\title{
Triangulum II. Not Especially Dense After All
}

\author{
Evan N. Kirby ${ }^{1}$, Judith G. Cohen ${ }^{1}$, Joshua D. Simon ${ }^{2}$, Puragra Guhathakurta ${ }^{3}$, Anders O. Thygesen ${ }^{1}$, and Gina E. Duggan ${ }^{1}$ \\ ${ }^{1}$ California Institute of Technology, 1200 E. California Boulevard, MC 249-17, Pasadena, CA 91125, USA \\ ${ }^{2}$ Observatories of the Carnegie Institution of Washington, 813 Santa Barbara Street, Pasadena, CA 91101, USA \\ ${ }^{3}$ UCO/Lick Observatory and Department of Astronomy and Astrophysics, University of California, 1156 High Street, Santa Cruz, CA 95064, USA \\ Received 2017 January 7; revised 2017 March 3; accepted 2017 March 8; published 2017 March 29
}

\begin{abstract}
Among the Milky Way satellites discovered in the past three years, Triangulum II has presented the most difficulty in revealing its dynamical status. Kirby et al. identified it as the most dark-matter-dominated galaxy known, with a mass-to-light ratio within the half-light radius of $3600_{-2100}^{+3500} M_{\odot} L_{\odot}^{-1}$. On the other hand, Martin et al. measured an outer velocity dispersion that is $3.5 \pm 2.1$ times larger than the central velocity dispersion, suggesting that the system might not be in equilibrium. From new multi-epoch Keck/DEIMOS measurements of 13 member stars in Triangulum II, we constrain the velocity dispersion to be $\sigma_{v}<3.4 \mathrm{~km} \mathrm{~s}^{-1}$ (90\% C.L.). Our previous measurement of $\sigma_{v}$, based on six stars, was inflated by the presence of a binary star with variable radial velocity. We find no evidence that the velocity dispersion increases with radius. The stars display a wide range of metallicities, indicating that Triangulum II retained supernova ejecta and therefore possesses, or once possessed, a massive dark matter halo. However, the detection of a metallicity dispersion hinges on the membership of the two most metalrich stars. The stellar mass is lower than galaxies of similar mean stellar metallicity, which might indicate that Triangulum II is either a star cluster or a tidally stripped dwarf galaxy. Detailed abundances of one star show heavily depressed neutron-capture abundances, similar to stars in most other ultra-faint dwarf galaxies but unlike stars in globular clusters.
\end{abstract}

Key words: galaxies: abundances - galaxies: dwarf - Local Group

Supporting material: machine-readable tables

\section{Introduction}

Dwarf galaxies present excellent opportunities for studying a multitude of aspects of galaxy formation and cosmology. First, dwarf galaxies are especially sensitive to stellar feedback. They have shallow gravitational potentials because they have little mass. As a result, supernovae and even winds from low-mass stars can redistribute the metals in the galaxy's gas (e.g., Larson 1974) and even expel metals from the galaxy (e.g., Dekel \& Woo 2003). The effect of feedback is readily apparent in the low average metallicities of dwarf galaxies (Skillman et al. 1989). Second, dwarf galaxies contain a great deal of dark matter. They exhibit mass-to-light ratios (M/Ls) of tens to thousands in solar units (Faber \& Lin 1983; Simon \& Geha 2007; Geha et al. 2009; Simon et al. 2011). The overwhelming dominance of dark matter relative to the luminous matter makes dwarf galaxies ideal targets for examining dark matter density profiles (Walker et al. 2007) and for searching for self-annihilation of the dark matter particle in gamma-rays (e.g., Drlica-Wagner et al. 2015a).

Measuring the dark matter content of dwarf galaxies requires precision and delicacy. Measuring even the total dark matter mass - not to mention the mass profile-is nuanced. The mass is measured by quantifying the stellar velocity dispersion. Smaller masses imply shallower gravitational potentials and slower stellar orbits. Measuring the dispersion in velocity of stars in an ultra-faint dwarf galaxy (UFD) is difficult because it

\footnotetext{
* The data presented herein were obtained at the W. M. Keck Observatory, which is operated as a scientific partnership among the California Institute of Technology, the University of California and the National Aeronautics and Space Administration. The Observatory was made possible by the generous financial support of the W. M. Keck Foundation.
}

is not much larger than the uncertainty in the measurement of a single star's radial velocity (Simon \& Geha 2007).

Many UFDs do not have more than a few stars on the red giant branch (RGB). Thus, the velocity dispersions must be measured from a sample containing mostly faint stars $(V \gtrsim 19)$, precluding high-resolution spectroscopy. Therefore, the velocity precision is limited by small samples, spectral resolution, and the random noise in the spectrum.

Numerous systematic errors further limit the velocity precision (see Simon \& Geha 2007, Sohn et al. 2007, or Kirby et al. 2015c for descriptions of some of these effects). For example, imaging spectrographs use slits to isolate stars. Displacement of the star from the center of the slit will cause a shift in the measured wavelengths - and hence velocitiesrelative to any reference spectrum that fills the slit, like an arc lamp. Another source of systematic uncertainty is the precision of the wavelength solution. The wavelengths can be known only as well as the reference, usually emission lines from an arc lamp. The mapping from pixel position to wavelength also assumes a model, such as a polynomial, which may be too simplistic for some spectrographs. Furthermore, flexure and thermal contraction of the spectrograph during the night could cause the wavelength solution to wander. Finally, radial velocities are measured by comparing the observed spectrum to a synthetic or empirical template spectrum. A mismatch in the shapes or strengths of absorption lines in the template spectrum could lead to an additional source of systematic uncertainty.

There is also one major astrophysical source of error in the velocity dispersion. Binary stars have orbital velocities that could exceed the velocity dispersion of the galaxy by a factor of 10 or more. Although the center of mass of the binary system will follow galactic orbits, the velocities of the 
individual binary components should not be used in the model of the dwarf galaxy's velocity dispersion. If a star in a binary system is erroneously interpreted as a single star, then its velocity may artificially inflate the measured velocity dispersion. Using red giants to measure the velocity dispersion is especially prone to undetected binaries because the red giant is probably much brighter than its companion. The binary companion could have already evolved past the giant phase into a white dwarf, or the companion might still be on the main sequence. For a $12 \mathrm{Gyr}$ population, like an ancient dwarf galaxy, only stars with masses within $\sim 1 \%$ of each other will be on the RGB simultaneously (e.g., Demarque et al. 2004). For other binary systems, the red giant is likely to be many times brighter than its companion. Therefore, the only way to determine the binarity of most red giants in dwarf galaxies is multi-epoch spectroscopy to search for variability in radial velocity.

The binary frequency in dwarf galaxies is not well known. Minor (2013) estimated the binary fraction of four classical dwarf spheroidal galaxies (dSphs) using spectroscopic data to be $46_{-9}^{+13} \%$, consistent with the Milky Way (MW) field population. However, he also found that the fraction could vary among dSphs. For example, the binary fraction in the Carina dSph $\left(14_{-5}^{+28} \%\right)$ was found to be inconsistent with the MW field population at $90 \%$ confidence. M.E. Spencer et al. (2017, in preparation) found a binary fraction of approximately $(30 \pm 10) \%$ in the Leo II dSph. They estimated that such a binary fraction in a UFD with a velocity dispersion of $2.0 \mathrm{~km} \mathrm{~s}^{-1}$ could spuriously inflate the velocity dispersion on average to $2.7 \mathrm{~km} \mathrm{~s}^{-1}$, leading to an $80 \%$ overestimate in the galaxy's mass. Unfortunately, the binarity of the stellar populations in UFDs is even less well measured than classical dSphs. From Hubble Space Telescope photometry, Geha et al. (2013) measured a binary fraction of $\left(47_{-14}^{+16} \%\right)$ in the Hercules UFD and $\left(47_{-17}^{+37} \%\right)$ in the Leo IV UFD. Only one UFD star (in Hercules) has a complete binary orbit (Koch et al. 2014). Other binaries are known in Boötes I (Koposov et al. 2011) and Boötes II (Ji et al. 2016d).

In this article, we critically examine the previously published spectroscopic properties of the Triangulum II UFD (Tri II), paying particular attention to the dispersion and spatial distribution of stellar radial velocities. Laevens et al. (2015) discovered Tri II at a distance of $30 \pm 2 \mathrm{kpc}$ in the Panoramic Survey Telescope and Rapid Response System (Pan-STARRS; Kaiser et al. 2010) photometric survey. Its luminosity is just $450 L_{\odot}$, and its 2D half-light radius is only 34 pc. Kirby et al. (2015a, hereafter K15a) measured its velocity dispersion from medium-resolution, multi-object spectroscopy to be $\sigma_{v}=5.1_{-1.4}^{+4.0} \mathrm{~km} \mathrm{~s}^{-1}$ from six stars near the galaxy's center. Martin et al. (2016, hereafter M16) corroborated this central velocity dispersion and found that it increased to as much as $14 \mathrm{~km} \mathrm{~s}^{-1}$ in the outermost parts of the galaxy. Such a galaxy would almost certainly be out of dynamical equilibrium. If Tri II is in dynamical equilibrium, either measurement of the velocity dispersion would make it the most dark-matterdominated galaxy known. As a result, it would be an excellent candidate for the indirect detection of dark matter from selfannihilation (Genina \& Fairbairn 2016).

The chemical properties of Tri II suggest a very metal-poor stellar system $(\langle[\mathrm{Fe} / \mathrm{H}]\rangle \approx-2.5)$. K15a found that the metallicities of three stars are inconsistent with being identical. As a result, Tri II chemically enriched itself, a hallmark of a bona fide galaxy rather than a star cluster (Willman \& Strader 2012). Venn et al. (2017, hereafter V17) additionally obtained high-resolution spectra of two stars in Tri II. The two stars differ in iron abundance by $(0.4 \pm 0.3)$ dex. The magnesium and calcium abundances are discrepant at $\sim 2 \sigma$. Thus, these two high-resolution spectra support K15a's claim that Tri II is chemically diverse.

We describe our observations in Section 2. Sections 3 and 4 detail our medium-resolution spectroscopic measurements of velocities and metallicities, respectively. We additionally obtained a high-resolution spectrum of one star, as described in Section 5. Section 6 closes with a discussion of our findings.

\section{Observations}

\subsection{Keck/DEIMOS}

The Deep Extragalactic Imaging Multi-Object Spectrograph (DEIMOS; Faber et al. 2003) is a mediumresolution spectrograph at the Nasmyth focus of the Keck II telescope. The multi-object mode employs custom-milled aluminum slitmasks designed for a specific pointing and position angle. Each target has a unique slit cut for it. Due to the limited target density, a single slitmask could not accommodate all of the stars identified as members by both K15a and M16. Therefore, the target lists for the slitmasks were not identical. We designed six different slitmasks observed on five different dates (see Table 1). The targets were selected from the union of the member lists of K15a and M16. Some additional stars known to be non-members were also targeted.

The spectrographic configuration was the same for all six slitmasks. We used the $1200 \mathrm{G}$ grating, which has a groove spacing of $1200 \mathrm{~mm}^{-1}$ and a blaze wavelength of $7760 \AA$. This grating provided a spectral resolving power of $R=7000$ at $8500 \AA$. The central wavelength was set to be $7800 \AA$, yielding a typical wavelength range of $6500-9000 \AA$, but the range varied from star to star depending on the exact location of the slit on the slitmask. We used the OG550 order-blocking filter to isolate the light diffracted from the first order of the grating. We used a quartz lamp for flat fielding and $\mathrm{Ne}, \mathrm{Ar}, \mathrm{Kr}$, and $\mathrm{Xe}$ arc lamps for wavelength calibration. DEIMOS's flexure compensation system provided extra wavelength stability.

We reduced the DEIMOS spectra using spec2d (Cooper et al. 2012; Newman et al. 2013), a data reduction pipeline built by the Deep Extragalactic Exploration Probe (DEEP) team. We used our own modifications to the pipeline (Kirby et al. 2015b, 2015c). These modifications included an improvement to the way in which sky lines are traced along the slit, and the extraction was improved by taking into account differential atmospheric refraction. The final products of the pipeline are flat-fielded, wavelength-calibrated, sky-subtracted spectra and their associated variance arrays.

We adopted photometry from the first data release of PanSTARRS (Chambers et al. 2016; Flewelling et al. 2016). Figure 1 shows the observed stars in the CMD and celestial coordinates. For the purposes of estimating effective temperatures and surface gravities (Section 4), we converted PanSTARRS magnitudes to the Sloan Digital Sky Survey (SDSS) system following Tonry et al. (2012). The figures and tables in this article present the un-transformed, native Pan-STARRS photometry. We corrected magnitudes for reddening and extinction using the dust maps of Schlegel et al. (1998). 
Table 1

DEIMOS Observations

\begin{tabular}{|c|c|c|c|c|c|c|}
\hline Slitmask & Targets & UT Date & Airmass & $\begin{array}{c}\text { Seeing } \\
\left({ }^{\prime \prime}\right)\end{array}$ & Exposures & $\begin{array}{l}\text { Exp. Time } \\
\text { (s) }\end{array}$ \\
\hline TriIIb $^{a}$ & 29 & 2015 Oct 8 & 1.4 & 0.9 & 3 & 3120 \\
\hline Trillc & 25 & 2015 Dec 14 & 1.1 & 0.9 & 4 & 7200 \\
\hline TriIId & 28 & 2016 Jan 29 & 1.1 & 0.9 & 4 & 5100 \\
\hline Trille & 25 & 2016 Jan 29 & 1.3 & 1.1 & 3 & 4803 \\
\hline Trillf & 21 & 2016 Jun 29 & 1.6 & 1.0 & 2 & 2700 \\
\hline TriIIg & 27 & 2016 Sep 7 & 1.1 & 0.7 & 3 & 3060 \\
\hline
\end{tabular}

Note.

${ }^{\text {a }}$ Observations by K15a.

In this article, we refer to the stars by the identification given by M16 except for star 128, which was not in M16's sample.

\subsection{Keck/HIRES}

We obtained Keck/HIRES spectra of star 40, the brightest known member of Tri II, on 2016 August 25. Figure 1(a) identifies star 40 in the CMD. This star is also the subject of the high-resolution spectroscopic analysis by V17. We obtained seven exposures of $1800 \mathrm{~s}$ each for a total exposure time of $3.5 \mathrm{hr}$. The seeing was $0{ }^{\prime \prime} 5$. We used the $7{ }^{\prime \prime} 0 \times 0$ " 86 slit, which provided a spectral resolving power of $R=49,000$ over the spectral range of 3933-8374 $\mathrm{A}$ with some inter-order gaps redward of $6410 \AA$.

We used a quartz lamp for flat fielding and a ThAr arc lamp for wavelength calibration. We reduced the raw frames into 1D spectra using version 5.2.4 of the MAKEE data reduction pipeline. ${ }^{4}$ This pipeline produces a flat-fielded, wavelengthcalibrated 1D spectrum and associated variance array for each exposure. We summed the seven individual spectra to produce a single spectrum. We also constructed the error spectrum by summing the individual error spectra in quadrature.

The HIRES spectrum is composed of 47 observed echelle orders, some of which overlap in wavelength. We combined the orders into one continuous spectrum except for the interorder gaps in the redder part of the spectrum. Before we could combine them, we needed to remove the response function, which caused the edges of orders to have fewer counts than the middle of orders. We fit a separate spline to each order. The splines had breakpoint spacings of 500 pixels. After dividing by the spline, the order appeared flat. We then interpolated all the orders onto a uniform wavelength array. We averaged overlapping parts of different orders with inverse variance weighting to maximize the $S / N$. We calculated the final spectrum's $\mathrm{S} / \mathrm{N}$ as the standard deviation of the continuumdivided spectrum in the relatively line-free spectral region from 5720 to $5800 \AA$. The result is $\mathrm{S} / \mathrm{N}=53 \mathrm{pixel}^{-1}$ or 103 per spectral resolution element.

\section{Kinematics}

We measured radial velocities from the DEIMOS spectra in the same manner as K15a and Kirby et al. (2015c). We refer the reader to those articles for more detail on the technique. Briefly, we matched the observed spectra to template spectra. We varied the radial velocity of the template spectra to minimize $\chi^{2}$. We also correcting for mis-centering in the slit by shifting

\footnotetext{
4 http://www.astro.caltech.edu/ tb/makee/
}

the spectrum to ensure that telluric features, such as the Fraunhofer A and B bands, were at zero velocity. We estimated velocity errors by re-measuring the radial velocity with the best-fitting template but with noise added to the observed spectrum. We took the random velocity uncertainty to be the standard deviation among 1000 noise trials. As found previously (Simon \& Geha 2007; Kirby et al. 2015c), the random noise is an incomplete description of the velocity error. We added a systematic error component of $1.49 \mathrm{~km} \mathrm{~s}^{-1}$ in quadrature with the random error to estimate the total error. Table 2 gives the coordinates, photometry, velocities, and membership of each star. The VI photometry is from the Keck/ LRIS observations by K15a, and the gi photometry is from the Pan-STARRS observations by M16. For stars observed multiple times, the velocity quoted is the mean of the individual observations weighted by the inverse variance. The velocities are in the heliocentric frame.

\subsection{Membership}

We used roughly the same membership criteria as K15a. K15a used a velocity cut based on the velocity dispersion. However, we show in Section 3.4 that we could not resolve the velocity dispersion of Tri II. Therefore, we accept as members any stars within $50 \mathrm{~km} \mathrm{~s}^{-1}$ of the mean velocity that also pass the other membership cuts. We also checked that no member star had a strong $\mathrm{Na}$ I $\lambda 8190$ doublet, which would indicate that the star is a foreground dwarf. These criteria did not rule out any star previously classified as a member by K15a and M16. The data set contains 13 stars identified as members by K15a and M16: 1 from K15a, 7 from M16, and 5 from both.

Additionally, we found that star 26 , with a velocity of $-375.6 \pm 11.2 \mathrm{~km} \mathrm{~s}^{-1}$, is a member. M16 measured $-84.1 \pm$ $8.2 \mathrm{~km} \mathrm{~s}^{-1}$ and therefore classified it as a non-member. Star 8 , classified as a member by M16, is not in our sample. Star 25 is a double-lined spectroscopic binary. Its DEIMOS spectrum has a bifurcated $\mathrm{H} \alpha$ absorption profile in exposures from both the TriIIc and Trilld slitmasks. The velocities of the individual $\mathrm{H} \alpha$ lines straddle the mean velocity of Tri II. Although it is probably a member of Tri II, we did not use it in the determination of the velocity dispersion because our incomplete temporal sampling did not allow us to determine the center-of-mass velocity reliably. The resulting sample size of candidate members (excluding star 25) is 13 .

Star 31 deserves some extra scrutiny. It has the highest metallicity $([\mathrm{Fe} / \mathrm{H}]=-1.40 \pm 0.13$; see Section 4) and largest separation from the center of the galaxy $\left(r=7 ! 6=2.0 r_{h}\right)$ of any of the member stars. It is also the only star whose $1 \sigma$ velocity error bar does not overlap the mean 

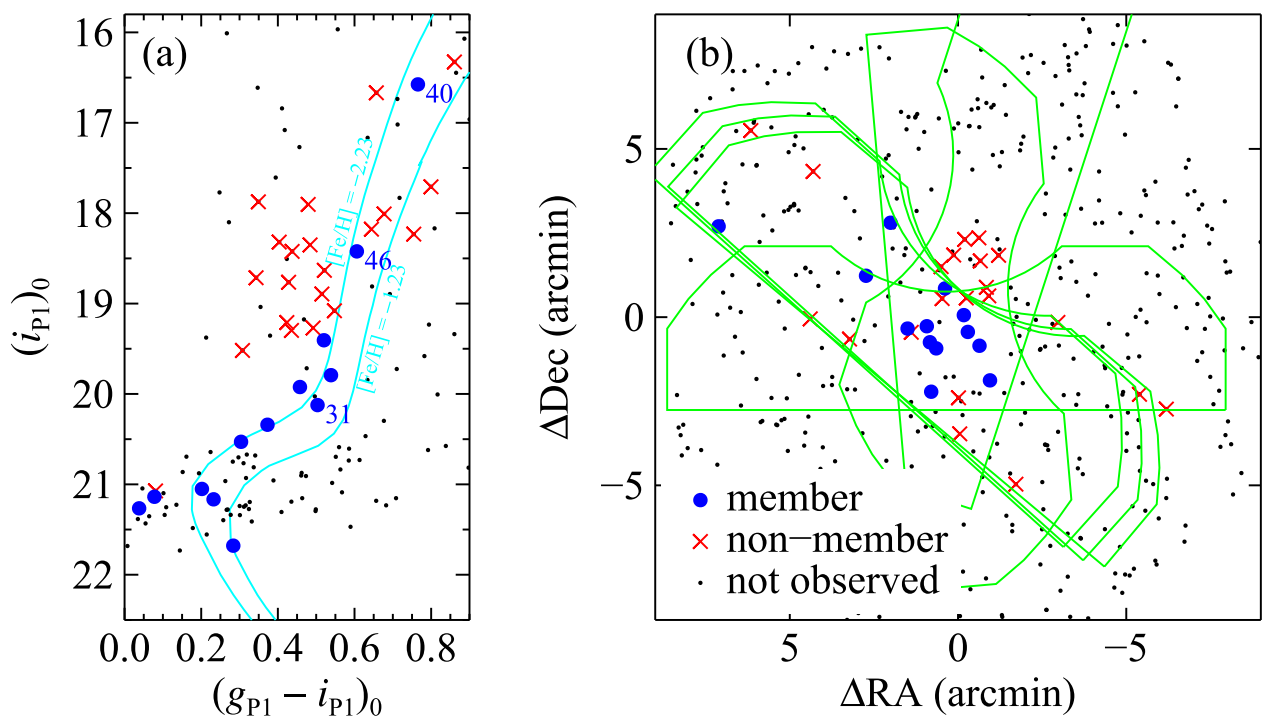

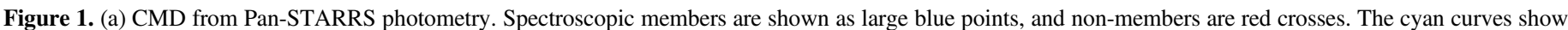

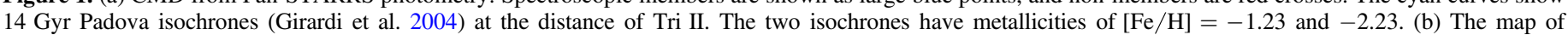
spectroscopic targets. The DEIMOS slitmask outlines are shown in green.

velocity of Tri II, but it is still consistent with expectations for a sample of 13 stars with Gaussian measurement uncertainties. We estimated the probability that star 31 is a non-member by querying the Besançon model of Galactic structure and kinematics (Robin et al. 2003). We selected only stars at the Galactic coordinates of Tri II with colors and magnitudes within 0.03 mag of star 31. In a large sample of synthetic stars, $0.47 \%$ had radial velocities within $6.1 \mathrm{~km} \mathrm{~s}^{-1}$ of Tri II's mean velocity, where $6.1 \mathrm{~km} \mathrm{~s}^{-1}$ is the sum of the $90 \%$ C.L. upper limit on $\sigma_{v}$ (calculated below) and the velocity uncertainty on star 31. In our sample of 21 stars classified as non-members by our DEIMOS spectroscopy, the probability of finding a non-member that falls in the velocity window is $1-(1-0.0047)^{21}=9.5 \%$. The CMD position of star 31 (Figure 1(a)) is consistent with its measured metallicity. The star also has a radial velocity and Na I $\lambda 8190$ line strength consistent with a red giant member of Tri II. Therefore, this star passes all of our membership criteria, and we retain it in our sample. We discuss its influence on the metallicity dispersion in Section 4.

\subsection{Validation and Radial Trends}

We compared our DEIMOS velocities with two other sources: our HIRES spectrum and M16. The HIRES velocity of star 40 is $-378.8 \pm 0.2 \mathrm{~km} \mathrm{~s}^{-1}$, which is $2.8 \pm 1.7 \mathrm{~km} \mathrm{~s}^{-1}$ higher than the DEIMOS velocity. There are several possible causes for the discrepancy. First, there is a 9\% chance that the difference arises from random noise, which is almost entirely dominated by the DEIMOS spectrum. Second, there could be a zeropoint offset in the wavelength calibration of HIRES versus DEIMOS. Third, we might have underestimated the error in the DEIMOS velocity measurement. Regardless, the discrepancy is not very large, and offsets of this magnitude do not affect our conclusions.

Our sample overlaps with that of M16 by 27 stars. We count 12 of these stars as members, excluding star 25, the doublelined spectroscopic binary. M16 did not consider one of these, star 26, a member because they measured a much different radial velocity than we did. Figure 2 shows our measurements of radial velocity for the remaining 11 stars compared to M16. We determined star 46 to exhibit a radial velocity that varies in time (Section 3.3). Among the remaining sample of 10 stars, our measurements of radial velocity for 6 stars overlap with M16's measurements within the quadrature sum of the $1 \sigma$ error bars. For perfectly estimated errors, we would have expected $68 \%$ of the sample, or about seven stars, to overlap. Another way to compare consistency between the samples is to examine the quantity $\left(v_{\mathrm{K} 17}-v_{\mathrm{M} 16}\right) / \sqrt{\delta v_{\mathrm{K} 17}^{2}+\delta v_{\mathrm{M} 16}^{2}}$. For perfect errors, this quantity would be normally distributed with a mean of $(0 \pm 1)$ and a standard deviation of $(1 \pm 0.18)$, assuming $N=10$. We measured a mean of -0.63 and a standard deviation of 0.93 . Thus, the samples are roughly consistent after excluding stars 25,26 , and 46 . However, as we show in Section 3.4, the consistency between the two samples does not guarantee that we infer the same properties of the underlying velocity distribution.

Our updated velocities affect M16's conclusion that the velocity dispersion increases with radius. Figure 3 shows our measurements of velocity versus angular distance from the center of Tri II. M16 claimed that the velocity dispersion increased from $4.4_{-2.0}^{+2.8} \mathrm{~km} \mathrm{~s}^{-1}$ in the central $2^{\prime}$ (blue shaded region) to $14.1_{-4.2}^{+5.8} \mathrm{~km} \mathrm{~s}^{-1}$ outside of $2^{\prime}$ (red shaded region). There is no apparent increase of velocity dispersion or change in mean velocity with radius in our data. In fact, the velocity of every member star in our sample except star 31 is consistent with the mean velocity within $1 \sigma$. We note that our exclusion of star 25, the double-lined spectroscopic binary, is partly responsible for our finding of a smaller velocity dispersion at $r>2^{\prime}$ than M16 found. However, our revisions to the velocities of the three stars mentioned in the previous paragraph are the primary cause for our downward revision of the outer velocity dispersion.

\subsection{Velocity Variability}

The advantage of observing stars over multiple epochs is that their radial velocities can be monitored. Any change in velocity in excess of the measurement uncertainty likely indicates binarity. Figure 4 shows the individual velocity measurements 
Table 2

Radial Velocities

\begin{tabular}{|c|c|c|c|c|c|c|c|c|c|c|c|c|c|}
\hline ID (K15a) & ID (M16) & R.A. (J2000) & Decl. (J2000) & $\begin{array}{l}\text { Radius } \\
\text { (arcmin) }\end{array}$ & $\begin{array}{l}\left(g_{\mathrm{P} 1}\right)_{0} \\
(\mathrm{mag})\end{array}$ & $\begin{array}{c}\delta g_{\mathrm{P} 1} \\
(\mathrm{mag})\end{array}$ & $\begin{array}{l}\left(i_{\mathrm{P} 1}\right)_{0} \\
(\mathrm{mag})\end{array}$ & $\begin{array}{c}\delta i_{\mathrm{P} 1} \\
(\mathrm{mag})\end{array}$ & Masks & $\begin{array}{l}\mathrm{S} / \mathrm{N}^{\mathrm{a}} \\
\left(\AA^{-1}\right)\end{array}$ & $\begin{array}{c}\begin{array}{c}v_{\text {helio }} \\
\left(\mathrm{km} \mathrm{s}^{-1}\right)\end{array}\end{array}$ & $\sigma(v)$ & Member? \\
\hline 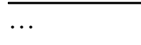 & 22 & $02 \quad 13 \quad 12.69$ & +360849.4 & 2.11 & 20.71 & 0.09 & 20.34 & 0.09 & cdefg & 44.5 & $-380.6 \pm 3.0$ & 0.6 & $\mathrm{Y}$ \\
\hline 128 & $\ldots$ & 021314.24 & +360951.1 & 1.06 & 19.93 & 0.03 & 19.41 & 0.03 & bdeg & 25.0 & $-383.3 \pm 1.8$ & 0.4 & $\mathrm{Y}$ \\
\hline 116 & 21 & $\begin{array}{lll}02 & 13 & 15.96\end{array}$ & +361015.8 & 0.53 & 20.38 & 0.02 & 19.92 & 0.02 & bcdeg & 26.4 & $-381.4 \pm 3.2$ & 0.9 & $\mathrm{Y}$ \\
\hline 106 & 40 & $02 \quad 13 \quad 16.55$ & +361045.8 & 0.19 & 17.34 & 0.01 & 16.58 & 0.01 & bdef & 219.5 & $-381.6 \pm 1.6$ & 0.4 & $\mathrm{Y}$ \\
\hline 91 & 20 & $02 \quad 13 \quad 19.32$ & +361133.3 & 0.93 & 20.33 & 0.03 & 19.79 & 0.03 & bcfg & 29.7 & $-380.1 \pm 4.9$ & 1.7 & $\mathrm{Y}$ \\
\hline 76 & 23 & 021320.61 & +360946.5 & 1.12 & 20.83 & 0.06 & 20.53 & 0.06 & bcg & 17.0 & $-385.2 \pm 4.2$ & 1.3 & $\mathrm{Y}$ \\
\hline$\ldots$ & 27 & 021321.35 & +360829.1 & 2.36 & 21.30 & 0.07 & 21.27 & 0.07 & $\mathrm{~cd}$ & 20.0 & $-376.8 \pm 11.7$ & 1.6 & $\mathrm{Y}$ \\
\hline 65 & 46 & 021321.54 & +360957.4 & 1.11 & 19.03 & 0.01 & 18.42 & 0.01 & bdfg & 81.8 & $-381.0 \pm 5.9$ & 3.0 & $\mathrm{Y}$ \\
\hline$\ldots$ & 24 & 021322.00 & +361025.9 & 0.97 & 21.22 & 0.07 & 21.14 & 0.07 & d & 17.8 & $-370.4 \pm 17.1$ & $\ldots$ & $\mathrm{Y}$ \\
\hline$\ldots$ & 26 & $02 \quad 1324.83$ & +361021.8 & 1.54 & 21.40 & 0.11 & 21.17 & 0.11 & $\mathrm{c}$ & 19.9 & $-375.6 \pm 11.2$ & $\ldots$ & $\mathrm{Y}$ \\
\hline$\ldots$ & 9 & $02 \quad 1327.33$ & +361330.5 & 3.45 & 21.25 & 0.10 & 21.05 & 0.10 & $\mathrm{~d}$ & 17.6 & $-387.6 \pm 7.7$ & $\ldots$ & $\mathrm{Y}$ \\
\hline$\cdots$ & 29 & 021330.95 & +361156.0 & 3.00 & 21.96 & 0.20 & 21.68 & 0.20 & $\mathrm{c}$ & 14.0 & $-386.2 \pm 4.7$ & $\ldots$ & $\mathrm{Y}$ \\
\hline$\cdots$ & 31 & 021352.66 & +361324.1 & 7.61 & 20.63 & 0.03 & 20.12 & 0.03 & cdg & 42.8 & $-377.1 \pm 2.7$ & 0.9 & $\mathrm{Y}$ \\
\hline$\ldots$ & 25 & $\begin{array}{lll}02 & 13 & 17.14\end{array}$ & +360714.1 & 3.47 & 21.15 & 0.05 & 21.07 & 0.05 & $\mathrm{~cd}$ & 21.3 & $\ldots$ & $\ldots$ & $?^{\mathrm{b}}$ \\
\hline$\ldots$ & 1 & 021246.71 & +360758.2 & 6.77 & 19.63 & 0.01 & 19.21 & 0.01 & $\mathrm{e}$ & 49.2 & $-85.4 \pm 1.8$ & $\ldots$ & $\mathrm{N}$ \\
\hline$\ldots$ & 2 & 021250.67 & +360824.6 & 5.86 & 19.76 & 0.02 & 19.27 & 0.02 & e & 55.0 & $-89.8 \pm 2.1$ & $\cdots$ & $\mathrm{N}$ \\
\hline$\ldots$ & 14 & 021302.68 & +361032.9 & 2.97 & 19.19 & 0.01 & 18.76 & 0.01 & $\mathrm{e}$ & 71.7 & $-40.6 \pm 1.6$ & $\ldots$ & $\mathrm{N}$ \\
\hline$\ldots$ & 34 & 021308.87 & +360544.3 & 5.25 & 18.38 & 0.01 & 17.90 & 0.01 & $\mathrm{~d}$ & 122.5 & $-40.7 \pm 1.5$ & $\ldots$ & $\mathrm{N}$ \\
\hline 166 & $\ldots$ & $02 \quad 13 \quad 11.42$ & +361232.4 & 2.21 & 18.51 & 0.04 & 17.71 & 0.04 & $\mathrm{~b}$ & 115.8 & $11.0 \pm 5.9$ & $\ldots$ & $\mathrm{N}$ \\
\hline 174 & $\ldots$ & $\begin{array}{lll}02 & 13 & 12.86\end{array}$ & +361120.1 & 1.12 & 18.22 & 0.01 & 17.87 & 0.01 & $\mathrm{~b}$ & 102.7 & $-100.8 \pm 1.6$ & $\ldots$ & $\mathrm{N}$ \\
\hline 177 & 4 & $02 \quad 13 \quad 13.22$ & +361135.4 & 1.23 & 19.83 & 0.01 & 19.52 & 0.01 & be & 36.7 & $-278.0 \pm 3.6$ & 1.4 & $\mathrm{~N}$ \\
\hline 126 & 15 & $02 \quad 13 \quad 14.11$ & +361222.5 & 1.80 & 17.19 & 0.00 & 16.33 & 0.00 & $\mathrm{~b}$ & 127.2 & $2.4 \pm 1.5$ & $\ldots$ & $\mathrm{N}$ \\
\hline 127 & $\cdots$ & $02 \quad 13 \quad 14.32$ & +361303.5 & 2.45 & 19.63 & 0.01 & 19.08 & 0.01 & $\mathrm{~b}$ & 54.5 & $-86.0 \pm 1.8$ & $\cdots$ & $\mathrm{N}$ \\
\hline 113 & 19 & $0213 \quad 16.18$ & +361116.3 & 0.62 & 19.15 & 0.01 & 18.63 & 0.01 & bdfg & 72.7 & $-56.0 \pm 2.7$ & 1.4 & $\mathrm{~N}$ \\
\hline 111 & $\cdots$ & 021316.41 & +361300.8 & 2.33 & 19.06 & 0.01 & 18.71 & 0.01 & bf & 66.7 & $-61.3 \pm 1.8$ & 0.5 & $\mathrm{~N}$ \\
\hline$\cdots$ & 47 & $\begin{array}{lll}02 & 13 & 17.33\end{array}$ & +360818.3 & 2.40 & 18.72 & 0.02 & 18.32 & 0.02 & cdefg & 96.7 & $-71.6 \pm 1.6$ & 0.4 & $\mathrm{~N}$ \\
\hline 100 & $\cdots$ & $0213 \quad 18.03$ & +36 1232.9 & 1.85 & 18.99 & 0.01 & 18.23 & 0.01 & bf & 87.2 & $-36.4 \pm 2.8$ & 1.5 & $\mathrm{~N}$ \\
\hline 84 & $\ldots$ & 021319.74 & +361115.3 & 0.72 & 19.41 & 0.01 & 18.89 & 0.01 & $\mathrm{~b}$ & 60.7 & $-66.4 \pm 1.7$ & $\ldots$ & $\mathrm{N}$ \\
\hline 82 & 44 & 021319.89 & +361212.3 & 1.58 & 18.69 & 0.01 & 18.01 & 0.01 & bf & 101.8 & $-176.9 \pm 1.5$ & 0.1 & $\mathrm{~N}$ \\
\hline 45 & 42 & 021324.28 & +361015.1 & 1.45 & 17.32 & 0.00 & 16.67 & 0.00 & $\mathrm{~b}$ & 153.0 & $-62.1 \pm 1.5$ & $\ldots$ & $\mathrm{N}$ \\
\hline$\cdots$ & 45 & 021333.37 & +361003.1 & 3.29 & 18.86 & 0.01 & 18.42 & 0.01 & cdeg & 113.9 & $-59.7 \pm 2.6$ & 1.4 & $\mathrm{~N}$ \\
\hline$\cdots$ & 3 & 021338.73 & +361502.0 & 6.10 & 19.73 & 0.02 & 19.30 & 0.02 & $\mathrm{cdg}$ & 56.3 & $-67.8 \pm 1.6$ & 0.2 & $\mathrm{~N}$ \\
\hline$\ldots$ & 50 & 021339.17 & +361039.3 & 4.39 & 18.83 & 0.00 & 18.35 & 0.00 & $\mathrm{e}$ & 83.5 & $-29.8 \pm 1.6$ & $\ldots$ & $\mathrm{N}$ \\
\hline$\cdots$ & 18 & 021347.91 & +361615.1 & 8.28 & 18.82 & 0.01 & 18.18 & 0.01 & $\mathrm{dg}$ & 95.2 & $-149.1 \pm 1.7$ & 0.6 & $\mathrm{~N}$ \\
\hline
\end{tabular}

Notes.

${ }^{\mathrm{a}}$ To convert to $\mathrm{S} / \mathrm{N}$ per pixel, multiply by 0.57 .

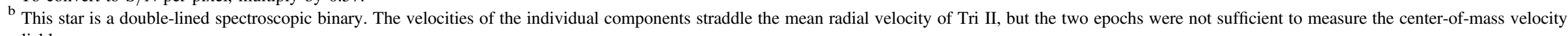
reliably.

(This table is available in machine-readable form.) 


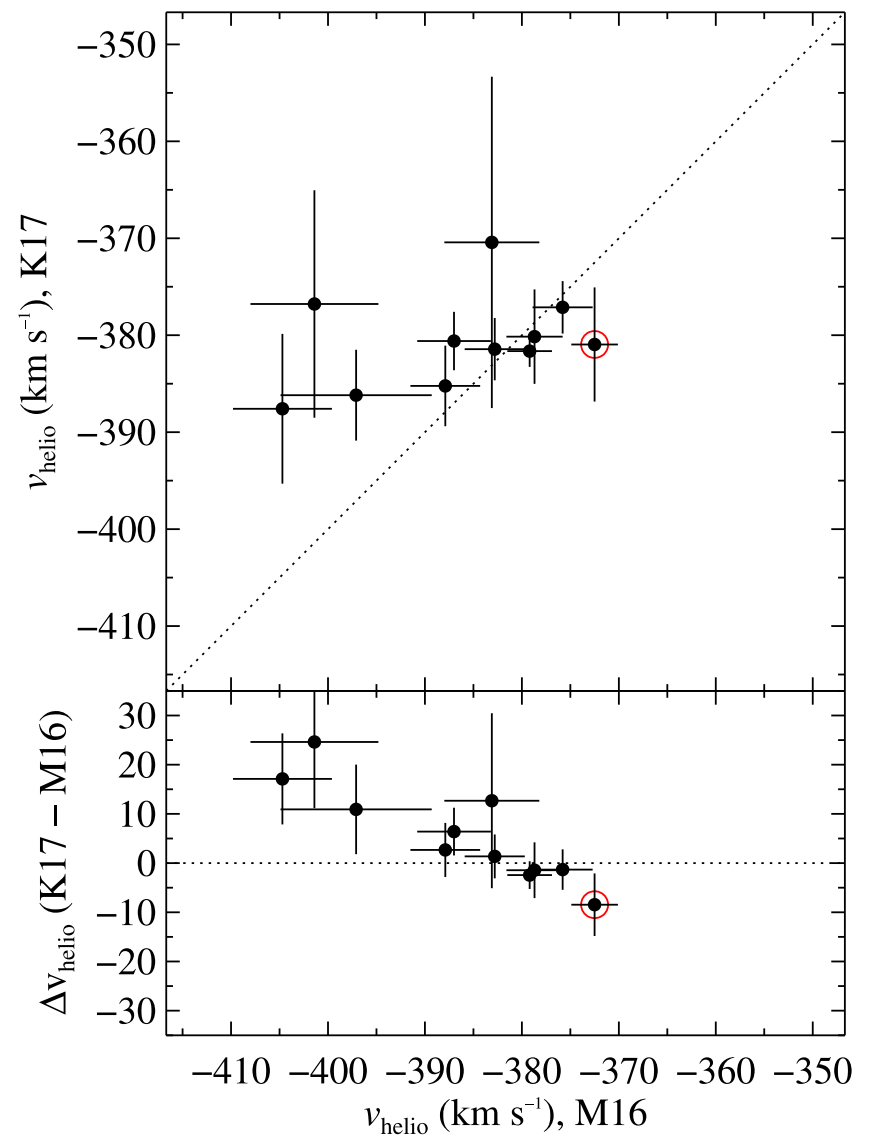

Figure 2. Velocity measurements from this work (K17) compared to M16. The dotted lines show where the two measurements would be equal. Star 46, the binary, is indicated by a red circle. The K17 value of the velocity for the star is the mean of our four measurements. Star 26 is not shown because M16 measured $v_{\text {helio }}=-84.1 \pm 8.2 \mathrm{~km} \mathrm{~s}^{-1}$, whereas we measured $v_{\text {helio }}=$ $-375.6 \pm 11.2 \mathrm{~km} \mathrm{~s}^{-1}$

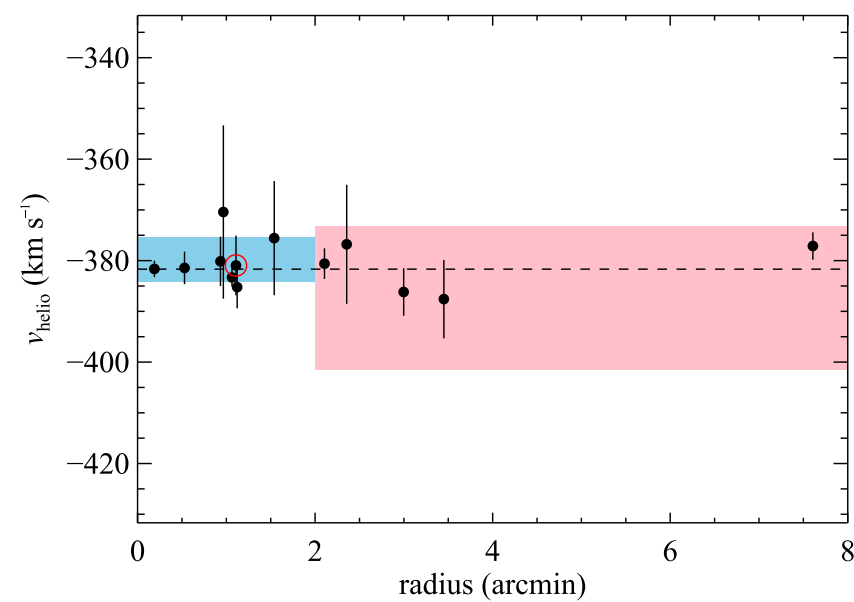

Figure 3. Radial velocity of Tri II members vs. their distances from the center of the galaxy. The dashed line shows the mean velocity. The shaded regions show the velocity dispersions in different regions of the galaxy published by M16. The mean velocity of star 46, the binary, is indicated by a red circle. Our measurements do not show evidence for a velocity dispersion changing with radius.

of the 12 member stars. Each column is the velocity curve of a unique member star. The horizontal spacing between measurements within a column is proportional to the time elapsed between observations.

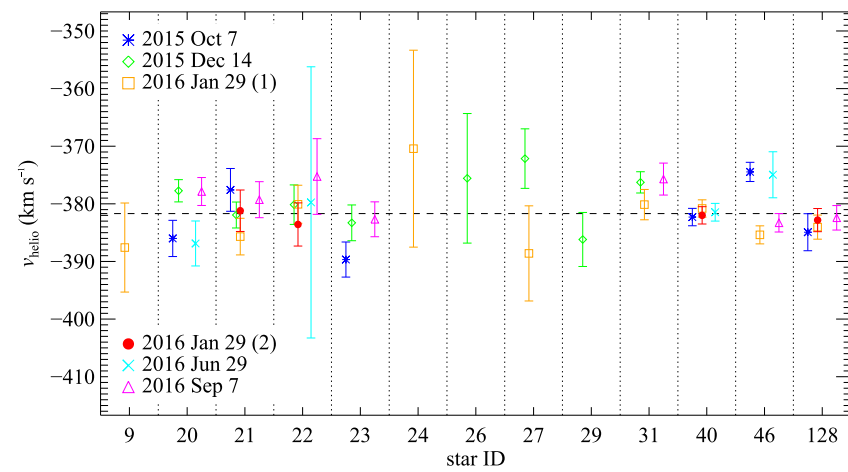

Figure 4. Heliocentric radial velocities observed at six different epochs. Each vertical column represents the measurements for one star. Within the column, the stars are ordered according to the date of observation. There are two independent measurements on 2016 January 26.

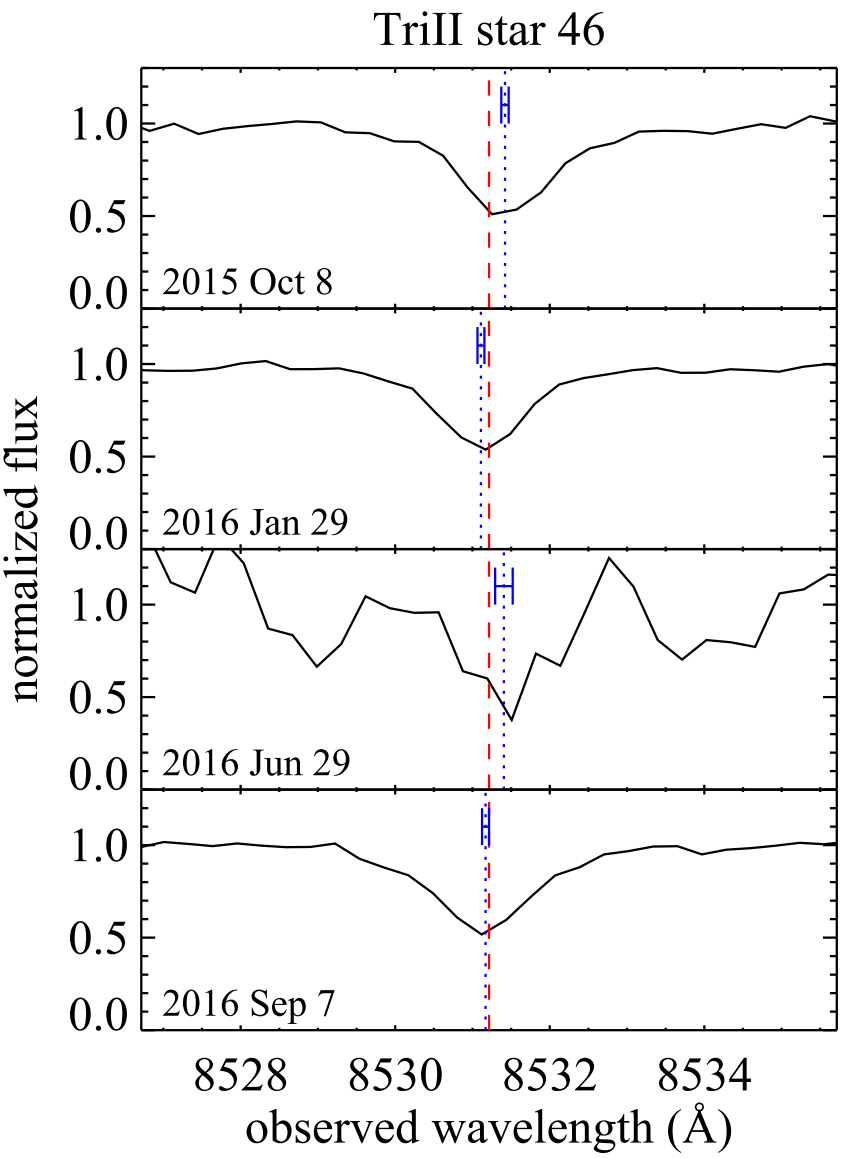

Figure 5. Four observations of star 46. Only a small region of the spectrum around Ca II $\lambda 8542$ is shown. The wavelengths are corrected for slit miscentering, and they are shown in the heliocentric frame. The red dashed line shows the expected center of the line at the mean velocity of Tri II: $\left\langle v_{\text {helio }}\right\rangle=-381.7 \mathrm{~km} \mathrm{~s}^{-1}$. The blue dotted lines show the center of the line at the measured velocity for each observation. The blue whiskers surrounding the blue dotted lines reflect the uncertainty in the position of the line center. The four observations show significant variation, indicating that the star's velocity is variable.

We quantified the velocity variability for each star as $\sigma(v)=\operatorname{stddev}\left(v_{i} / \delta v_{i}\right)$. Only star 46 had $\sigma(v)>2$. Figure 5 demonstrates the shift in the observed wavelength of Ca II $\lambda 8542$ in star 46 for four different dates. The changing wavelength is apparent even by eye. V17 also identified star 46 as a velocity variable from high-resolution spectroscopy. 
However, their measurement of the velocity amplitude $\left(24.5 \pm 2.1 \mathrm{~km} \mathrm{~s}^{-1}\right) \quad$ is significantly higher than the $10.9 \mathrm{~km} \mathrm{~s}^{-1}$ peak-to-peak amplitude that we observed with DEIMOS. Our temporal sampling is not dense enough to identify the true radial velocity amplitude with any certainty. V17's third of three velocity measurements for star 46 occurred between the dates of our first and second measurements. It is possible that the star's orbit is such that V17 measured the velocity close to the peak, whereas our measurements happened to be spaced closer to the midpoint of the velocity curve.

Our four DEIMOS radial velocity samples of star 46 are consistent with a period of $\sim 220$ days and a semi-amplitude of $\sim 5 \mathrm{~km} \mathrm{~s}^{-1}$. Assuming an edge-on, circular orbit and a primary mass of $0.8 M_{\odot}$, the mass of the secondary and the separation are uniquely constrained to be $0.14 M_{\odot}$ and 0.7 au. Decreasing the inclination $(i<90)$ would increase the secondary mass and slightly increase the separation. If the semi-amplitude is instead $12 \mathrm{~km} \mathrm{~s}^{-1}$, as indicated by V17, then the secondary mass and separation would be $0.38 M_{\odot}$ and 0.8 au for an edge-on system. These ranges of mass ratios and separations are well within the known ranges for stellar multiplicity in the solar neighborhood (Duchêne \& Kraus 2013).

\subsection{Velocity Dispersion}

Because K15a measured radial velocities during only one epoch, we did not know that star 46-one of only six member stars in that article-was variable in velocity. K15a pointed out that removing this star (called star 65 in that article) resulted in a velocity dispersion of $\sigma_{v}=2.8_{-1.7}^{+4.0} \mathrm{~km} \mathrm{~s}^{-1}$ based on five stars. This is a marginally resolved velocity dispersion. Thus, the binary orbital velocity of star 46 was driving the measurement of the velocity dispersion of Tri II rather than orbits in the galaxy's potential. In other words, star 46 was responsible for K15a's erroneously high value of $\sigma_{v}$.

Here, we revise our estimate of $\sigma_{v}$, taking into account the newly discovered binarity of star 46 and the expanded sample of member stars with radial velocities. We re-measured velocity dispersion in the same manner as K15a, excluding star 46. For stars with multiple measurements, we took the average of $v_{\text {helio }}$ weighted by the inverse variance of the individual measurements. Our method of measuring $\sigma_{v}$ is based on Walker et al. (2006) and is described fully by K15a and Kirby et al. (2015c). Briefly, we define a likelihood function (Equation (1) of Kirby et al. 2015c) that the velocity measurements and their errors, $\left(v_{\text {helio }}\right)_{i}$ and $\left(\delta v_{\text {helio }}\right)_{i}$, are described by a mean velocity, $\left\langle v_{\text {helio }}\right\rangle$, and a dispersion, $\sigma_{v}$. We maximized the likelihood with a Monte Carlo Markov Chain (MCMC) with $10^{7}$ links. We used a Metropolis-Hastings algorithm to perform the optimization.

The top panel of Figure 6 shows the distribution of $\sigma_{v}$ in the $10^{7}$ MCMC trials. The distribution rises all the way to zero velocity dispersion. Thus, we could not resolve a velocity dispersion of Tri II. Instead, we estimated its upper limit. The $90 \%$ C.L. upper limit, enclosing $90 \%$ of the MCMC trials, is $\sigma_{v}<3.4 \mathrm{~km} \mathrm{~s}^{-1}$. The 95\% C.L. upper limit is $\sigma_{v}<4.2 \mathrm{~km} \mathrm{~s}^{-1}$. These values are also reported in Table 3. Tri II joins Segue 2, Boötes II, and Tucana III in the group of UFDs with spectroscopy of multiple stars but without a detection of the velocity dispersion (Kirby et al. 2013a; Ji et al. 2016d; Simon et al. 2016). These upper limits are consistent with K15a's value of $\sigma_{v}$ when star 46 is removed from the sample. The MCMC distribution of $\sigma_{v}$ from that sample of just five stars is
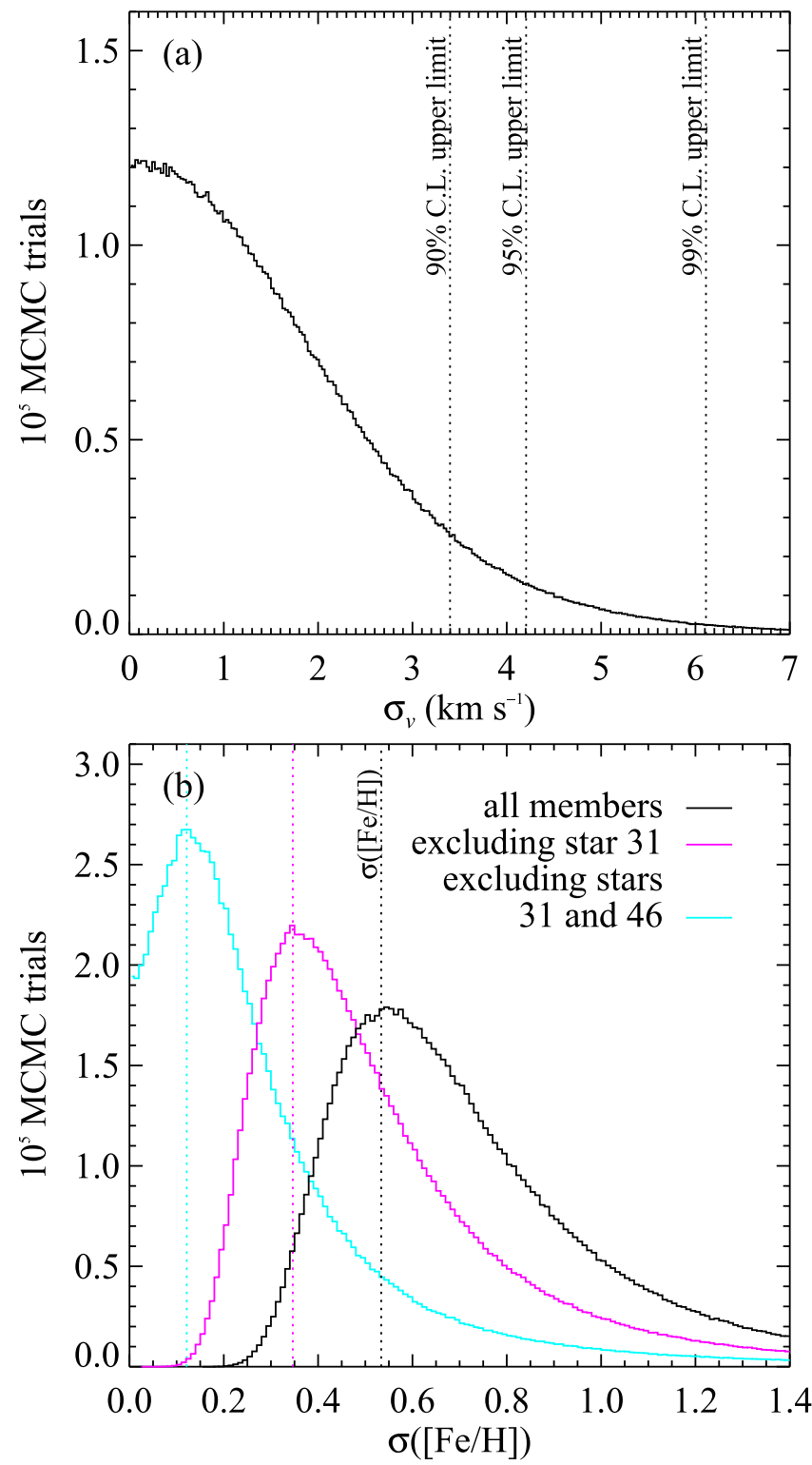

Figure 6. Posterior probability distribution on (a) the velocity dispersion and (b) metallicity dispersion. The histograms show the number of accepted MCMC trials in bins of $0.03 \mathrm{~km} \mathrm{~s}^{-1}$ for $\sigma_{v}$ and $0.01 \operatorname{dex}$ for $\sigma([\mathrm{Fe} / \mathrm{H}])$. The vertical dashed lines indicate confidence levels for the upper limit on $\sigma_{v}$ in the top panel and the mean value for $\sigma([\mathrm{Fe} / \mathrm{H}])$ in the bottom panel. The metallicity dispersion is computed for all candidate member stars (black), excluding star 46 (magenta), and excluding both star 46 and star 31 (cyan). Star 46 is a likely member, but it is a binary and very $\alpha$-poor. Star 31 is far from the galaxy center, and it is the most metal-rich star in the sample, but it passes every membership cut (Section 3.1).

not significantly distinct from zero. Thus, neither we nor K15a can resolve the velocity dispersion of Tri II.

The mass within the half-light radius of a spheroidal galaxy is proportional to the square of the velocity dispersion. Therefore, the downward revision to $\sigma_{v}$ implies a downward revision to the mass within the half-light radius of Tri II, which K15a estimated to be $M_{1 / 2}=\left(8.9_{-3.0}^{+6.8}\right) \times 10^{5} M_{\odot}$. In fact, we can estimate only an upper limit to the mass. Using Wolf et al.'s (2010) formula for dynamical mass, we constrain the mass within the 3D halflight radius to be $M_{1 / 2}<3.7 \times 10^{5} M_{\odot}(90 \% \quad$ C.L. $)$ or $M_{1 / 2}<5.6 \times 10^{5} M_{\odot}(95 \%$ C.L. $)$. 
Table 3

Properties of Triangulum II

\begin{tabular}{ll}
\hline \hline Property & Value \\
\hline$N_{\text {member }}$ & 13 \\
$\log \left(L_{V} / L_{\odot}\right)$ & $2.65 \pm 0.20$ \\
$r_{h}$ & $3.9_{-0.9}^{+1.1} \operatorname{arcmin}$ \\
$r_{h}$ & $34_{-8}^{+9} \mathrm{pc}$ \\
$\left\langle v_{\text {helio }}\right\rangle$ & $-381.7 \pm 1.1 \mathrm{~km} \mathrm{~s}^{-1}$ \\
$v_{\mathrm{GSR}}$ & $-261.7 \mathrm{~km} \mathrm{~s}^{-1}$ \\
$\sigma_{v}$ & $<3.4 \mathrm{~km} \mathrm{~s}^{-1}(90 \%$ C.L. $)$ \\
& $<4.2 \mathrm{~km} \mathrm{~s}^{-1}(95 \%$ C.L. $)$ \\
$M_{1 / 2}$ & $<3.7 \times 10^{5} \mathrm{~km} \mathrm{~s}^{-1}(90 \%$ C.L. $)$ \\
$\left(M / L_{V}\right)_{1 / 2}{ }^{\mathrm{a}}$ & $<5.6 \times 10^{5} \mathrm{~km} \mathrm{~s}^{-1}(95 \%$ C.L. $)$ \\
& $<1640 M_{\odot} L_{\odot}^{-1}(90 \%$ C.L. $)$ \\
$\rho_{1 / 2}{ }^{\mathrm{b}}$ & $<2510 M_{\odot} L_{\odot}^{-1}(95 \%$ C.L. $)$ \\
$\langle[\mathrm{Fe} / \mathrm{H}]\rangle$ & $<2.2 M_{\odot} \mathrm{pc}^{-3}(90 \%$ C.L. $)$ \\
\end{tabular}

Notes. The measurements of $\log L_{V}$ and $r_{h}$ come from Laevens et al. (2015). a Mass-to-light ratio within the half-light radius, calculated as $M_{1 / 2}=4 G^{-1} \sigma_{v}^{2} r_{h}$ (Wolf et al. 2010).

${ }^{\mathrm{b}}$ Density within the half-light radius.

Our sample overlaps M16's sample by 10 member stars after excluding stars 25,26 , and 46 . We repeated the estimation of $\sigma_{v}$ on M16's measurements of velocities for these 10 stars. We resolved the velocity dispersion as $\sigma_{v}=9.1_{-2.0}^{+3.9} \mathrm{~km} \mathrm{~s}^{-1}$, consistent with M16's estimation. Therefore, the different result obtained from our sample versus M16's sample is not strictly a result of the exclusion of certain stars. Instead, our higher- $\mathrm{S} / \mathrm{N}$ velocity measurements themselves are different enough from M16 to reduce $\sigma_{v}$ to an undetectable level.

This new mass limit indicates that Tri II does not have the largest $M / L$ of any non-disrupting galaxy. Instead, the upper limit on $M / L$ is consistent with the envelope of $M / L$ versus luminosity defined by other dwarf galaxies (Figure 7(c)). Furthermore, the amount of dark matter in Tri II is less than previously estimated. As a result, Tri II is not likely to be a significantly better candidate for indirect dark matter detection than other UFDs of similar luminosity.

\section{Metallicity and $[\alpha / \mathrm{Fe}]$}

In addition to radial velocities, we also measured metallicities and detailed abundances from the DEIMOS spectra. To maximize $\mathrm{S} / \mathrm{N}$ for abundance measurements, we coadded the 1D spectra of the nine member stars that had multiple observations. For star 46, we removed the Doppler shift due to the binary orbital velocity before coaddition. We measured atmospheric parameters for the stars using spectral synthesis, as described by Kirby et al. $(2008,2010)$. We used the known distance, $30 \pm 2 \mathrm{kpc}$ (Laevens et al. 2015), in combination with Padova isochrones (Girardi et al. 2004) to determine the stars' surface gravities and a first guess at their effective temperatures $\left(T_{\text {eff }}\right)$. We measured $T_{\text {eff }},[\mathrm{Fe} / \mathrm{H}],[\mathrm{Mg} / \mathrm{Fe}]$, $[\mathrm{Si} / \mathrm{Fe}],[\mathrm{Ca} / \mathrm{Fe}],[\mathrm{Ti} / \mathrm{Fe}]$, and $[\alpha / \mathrm{Fe}]$ by fitting the observed, continuum-divided spectra to a grid of synthetic spectra. The ratio $[\alpha / \mathrm{Fe}]$ is not an arithmetic average of the ratios of individual $\alpha$ elements. Instead, it is measured by minimizing the $\chi^{2}$ of the fit to all $\alpha$ element absorption lines simultaneously. Table 4 lists the results for the six stars for

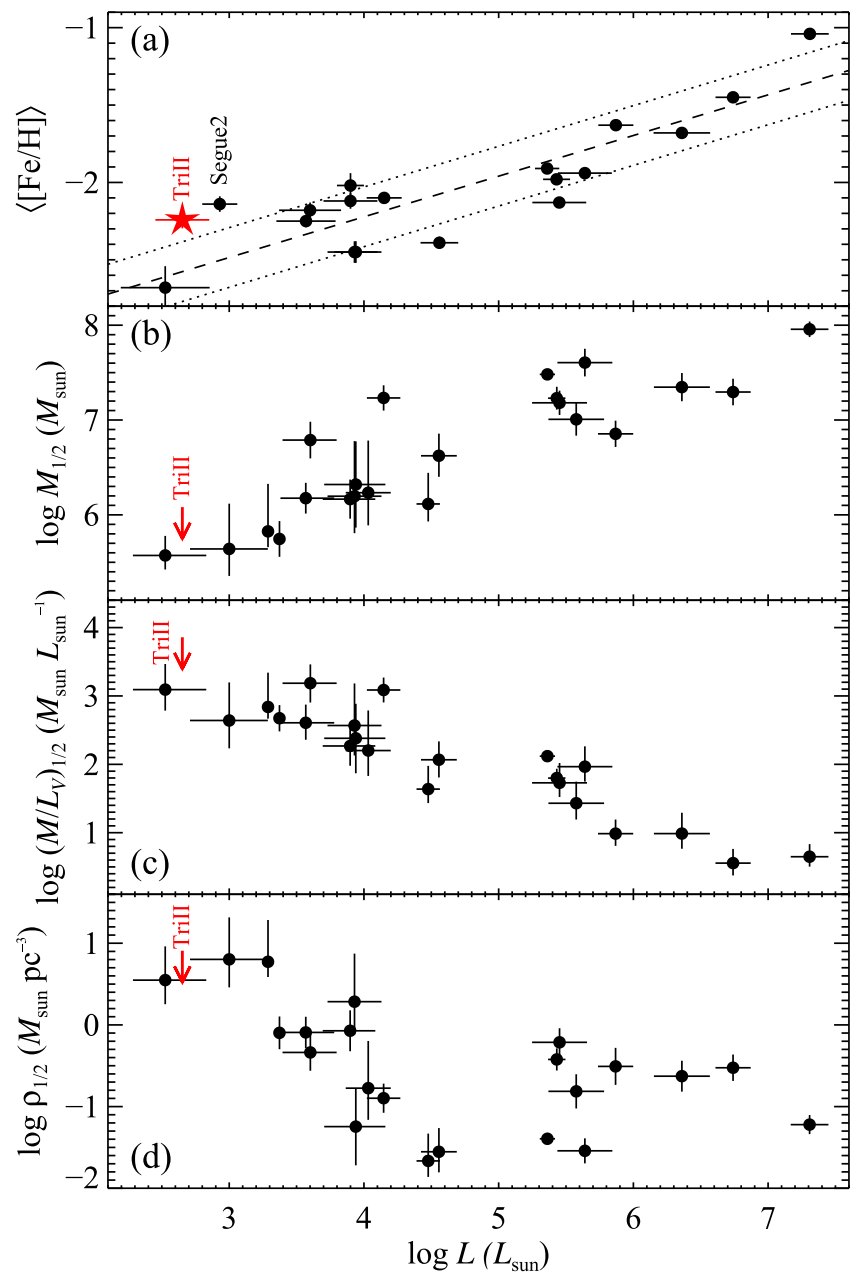

Figure 7. (a) Luminosity-metallicity relation. The dashed line shows the linear fit to the galaxies except Tri II (Kirby et al. 2013b, 2015c), and the dotted lines show the rms dispersion about the fit. (b) Masses of MW satellite galaxies within their 3D half-light radii assuming dynamical equilibrium. Data are from McConnachie (2012, and references therein), Simon et al. (2015), Koposov et al. (2015), and Kirby et al. (2015c). (c) Mass-to light ratios within the 3D half-light radii. (d) Mass densities within the 3D half-light radii.

which it was possible to measure abundances. The solar reference scale is from Asplund et al. (2009). ${ }^{5}$ The other member stars were too faint or their lines were too weak due to their warm temperatures. Table 3 gives the mean of the stellar metallicities weighted by their inverse variances.

We also measured metallicities from the near-infrared $\mathrm{Ca}$ triplet (CaT) following Starkenburg et al. (2010). This method uses the strength of Ca II $\lambda 8498$ and Ca II $\lambda 8542$ coupled with the absolute magnitude of the star (as a proxy for the temperature and gravity of the star) to estimate the star's metallicity. The calibration is based on iron abundance, not calcium abundance. Therefore, the CaT metallicities are called $[\mathrm{Fe} / \mathrm{H}]_{\mathrm{CaT}}$. Table 4 includes these measurements alongside the synthesis measurements. The CaT metallicities agree within 0.5 dex of the spectral synthesis except for star 46 . The discrepancy for that star could be due to its low $[\alpha / \mathrm{Fe}]$ ratio. The star could very well have an iron abundance consistent with the synthesis measurement and a calcium abundance consistent with the CaT measurement. In support of this

\footnotetext{
$\overline{12+\log n(\mathrm{Mg}) / n(\mathrm{H}) \equiv \epsilon(\mathrm{Mg})=7.58,}, \quad \epsilon(\mathrm{Si})=7.55, \quad \epsilon(\mathrm{Ca})=6.36$, $\epsilon(\mathrm{Ti})=4.99$, and $\epsilon(\mathrm{Fe})=7.50$.
} 
Table 4

Chemical Abundances

\begin{tabular}{|c|c|c|c|c|c|c|c|c|c|c|}
\hline ID (K15a) & ID (M16) & $\begin{array}{l}T_{\text {eff }} \\
(\mathrm{K})\end{array}$ & $\left(\begin{array}{ll}\log g \\
\left(\mathrm{~cm} \mathrm{~s}^{-2}\right)\end{array}\right.$ & {$[\mathrm{Fe} / \mathrm{H}]_{\mathrm{CaT}}$} & {$[\mathrm{Fe} / \mathrm{H}]$} & {$[\mathrm{Mg} / \mathrm{Fe}]$} & {$[\mathrm{Si} / \mathrm{Fe}]$} & {$[\mathrm{Ca} / \mathrm{Fe}]$} & {$[\mathrm{Ti} / \mathrm{Fe}]$} & {$[\alpha / \mathrm{Fe}]$} \\
\hline 128 & $\ldots$ & 5466 & 3.17 & $-3.01 \pm 0.08$ & $-2.44 \pm 0.15$ & $+0.46 \pm 0.83$ & $\ldots$ & $+0.39 \pm 0.22$ & $\ldots$ & $+0.08 \pm 0.28$ \\
\hline 116 & 21 & 5635 & 3.41 & $-3.14 \pm 0.13$ & $-2.86 \pm 0.21$ & $+1.07 \pm 0.67$ & $+0.59 \pm 0.50$ & $+0.24 \pm 0.36$ & $+1.00 \pm 0.44$ & $+0.42 \pm 0.36$ \\
\hline 106 & 40 & 4917 & 1.89 & $-2.71 \pm 0.02$ & $-2.78 \pm 0.11$ & $+0.59 \pm 0.38$ & $+0.43 \pm 0.17$ & $+0.45 \pm 0.12$ & $+0.27 \pm 0.14$ & $+0.40 \pm 0.10$ \\
\hline 91 & 20 & 5434 & 3.33 & $-2.13 \pm 0.06$ & $-2.53 \pm 0.17$ & $\ldots$ & $+0.50 \pm 0.35$ & $-0.27 \pm 0.83$ & $+0.71 \pm 0.42$ & $+0.16 \pm 0.33$ \\
\hline 65 & 46 & 5282 & 2.74 & $-2.56 \pm 0.04$ & $-1.91 \pm 0.11$ & $+0.21 \pm 0.28$ & $\ldots$ & $-0.39 \pm 0.15$ & $-0.79 \pm 0.76$ & $-0.48 \pm 0.15$ \\
\hline$\ldots$ & 31 & 5551 & 3.49 & $-0.90 \pm 0.07$ & $-1.40 \pm 0.13$ & $+0.89 \pm 0.26$ & $-0.09 \pm 0.28$ & $-0.37 \pm 0.25$ & $\ldots$ & $-0.28 \pm 0.23$ \\
\hline
\end{tabular}


hypothesis, the synthesis value of $[\mathrm{Ca} / \mathrm{H}]$ agrees well with $[\mathrm{Fe} / \mathrm{H}]_{\mathrm{CaT}}$.

Figure 7(a) shows the mass-metallicity relation for MW dwarf satellite galaxies (Kirby et al. 2013b, 2015c). The mean metallicity of Tri II lies above other UFDs of similar stellar mass. Equivalently, it could be said that Tri II is less massive than dwarf galaxies of similar metallicity. Interpreted in that way, it is easy to see how Tri II could have been tidally stripped by the MW. If the galaxy was originally more massive and it obeyed the mass-metallicity relation, like nearly every other satellite galaxy, then tidal stripping would remove mass but leave the mean metallicity nearly untouched. Thus, stripping corresponds to a leftward move in Figure 7(a). Segue 2 is another galaxy that appears to have been tidally stripped (Kirby et al. 2013a). Like Tri II, Segue 2 has an unresolved velocity dispersion, and its stellar mass is lower than most other galaxies of similar metallicity.

We computed the metallicity dispersion in the same manner as the velocity dispersion. We computed the likelihood (Equation (2) of Kirby et al. 2015c) that a mean metallicity and metallicity dispersion were consistent with our six measurements of $[\mathrm{Fe} / \mathrm{H}]$ and their errors. We used an MCMC with $10^{7}$ links to optimize the likelihood and hence to estimate $\sigma([\mathrm{Fe} / \mathrm{H}])=0.53_{-0.12}^{+0.38}$. The bottom panel of Figure 6 shows the probability distribution, which is a histogram of the accepted MCMC trials.

The metallicity dispersion is largely driven by star 31 and star 46 , the binary. If both are excluded, then the dispersion is only marginally resolved, as shown by the cyan probability distribution in Figure 6. An unresolved metallicity dispersion combined with an unresolved velocity dispersion would jeopardize Tri II's classification as a galaxy because a dispersion in at least one of those quantities is required to show that the stellar system has dark matter (Willman \& Strader 2012).

Star 31 shows no apparent reason to suspect a spurious metallicity measurement. Star 31's metallicity measured from the coadded spectrum, $[\mathrm{Fe} / \mathrm{H}]=-1.40 \pm 0.13$, agrees with the $[\mathrm{Fe} / \mathrm{H}]$ measurements from the individual spectra: $-1.29 \pm 0.20,-1.41 \pm 0.15$, and $-1.42 \pm 0.16$. Thus, there is no apparent problem in any individual spectrum that might lead to an anomalous measurement of metallicity. The only questionable aspect of star 31 is its membership (due to its distance from the center of the galaxy, its high metallicity, and its $1.6 \sigma$ deviation from the mean velocity).

Star 46 deserves some scrutiny for its unusually low $[\alpha / \mathrm{Fe}]=-0.48 \pm 0.15$. This value, measured from the coadded spectrum, agrees with the weighted mean of the individual spectra: $[\alpha / \mathrm{Fe}]=-0.51 \pm 0.27$. The metallicity from the coadded spectrum, $[\mathrm{Fe} / \mathrm{H}]=-1.91 \pm 0.11$, also agrees with the weighted mean: $[\mathrm{Fe} / \mathrm{H}]=-1.90 \pm 0.09$. The spectrum could be contaminated by the binary companion, but if the companion truly is an M dwarf (Section 3.3), then it is unlikely that the faint companion would affect the spectrum of the binary system in any measurable way.

V17 measured chemical abundances of star 46 from a low$\mathrm{S} / \mathrm{N}$, high-resolution spectrum. They measured $[\mathrm{Fe} / \mathrm{H}]=$ $-2.5 \pm 0.2$, which is inconsistent with our measurement of $[\mathrm{Fe} / \mathrm{H}]=-1.91 \pm 0.11$. One possible reason for the discrepancy is that we used ATLAS9 model atmospheres computed with an $[\alpha / \mathrm{Fe}]$ ratio consistent with the value measured from the spectrum (Kirby et al. 2010; Kirby 2011), whereas V17 used

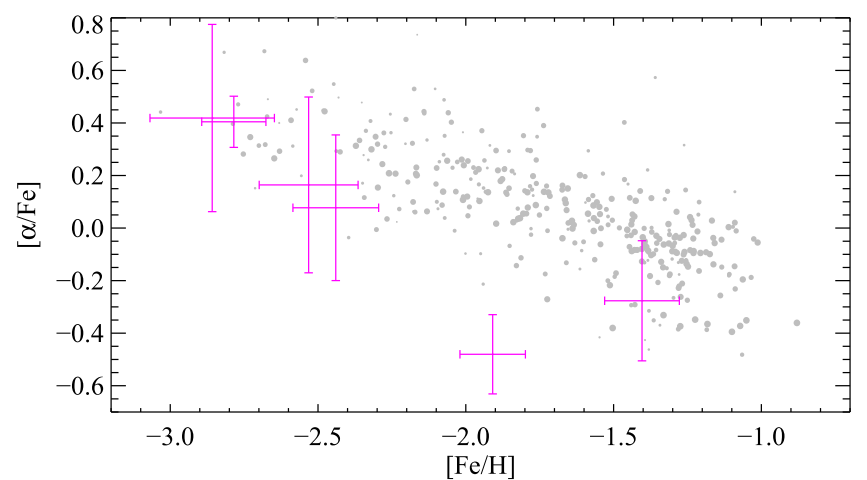

Figure 8. Trend of $[\alpha / \mathrm{Fe}]$ with metallicity in Tri II (magenta) compared to the larger dwarf galaxy Sculptor (gray; Kirby et al. 2009). The point sizes for Sculptor are inversely proportional to measurement uncertainty.

spherical MARCS model atmospheres (Gustafsson et al. 2008) with solar-scaled abundances. They also measured low $[\alpha / \mathrm{Fe}]$ $([\mathrm{Mg} / \mathrm{Fe}]=-0.7 \pm 0.3$ and $[\mathrm{Ca} / \mathrm{Fe}]=-0.2 \pm 0.3)$. If confirmed, these abundances would establish a dispersion in chemical abundance in Tri II, but it would not necessarily indicate selfenrichment by supernovae. Variations in light element abundances, especially $\mathrm{Mg}$, are well known in globular clusters (e.g., Gratton et al. 2004). Hence, if our measurement of $[\mathrm{Fe} / \mathrm{H}]$ for star 46 is erroneous and star 31 is a non-member, then Tri II could very well be a globular cluster.

Figure 8 shows the evolution of $[\alpha / \mathrm{Fe}]$ with increasing $[\mathrm{Fe} / \mathrm{H}]$. The decrease of $[\alpha / \mathrm{Fe}]$ is consistent with chemical evolution dominated by Type Ia supernovae ( $\mathrm{SNe}$ Ia) at late times. SNe Ia produce a great deal of iron and very little $\alpha$ elements, thereby depressing $[\alpha / \mathrm{Fe}]$ as $[\mathrm{Fe} / \mathrm{H}]$ increases. The decline in $[\alpha / \mathrm{Fe}]$ in Tri II is even more severe than in Sculptor, a larger dSph (gray points in Figure 8). The low $[\alpha / \mathrm{Fe}]$ ratios of stars 31 and 46 indicate that the galaxy's late-time chemical evolution was dominated by $\mathrm{SNe}$ Ia. Predictions of the nucleosynthetic yield of SNe II (e.g., Woosley \& Weaver 1995; Nomoto et al. 2006) have significantly larger $[\alpha / \mathrm{Fe}]$. The steep decline of $[\alpha / \mathrm{Fe}]$ in Tri II is similar to other UFDs (Vargas et al. 2013). The low $[\alpha / \mathrm{Fe}]$ ratio of star 46 is only slightly lower than stars of similar metallicity in the Hercules UFD. However, it is worth repeating that these conclusions hinge on either the accuracy of our measurement of $[\mathrm{Fe} / \mathrm{H}]$ for star 46 or the membership of star 31 .

\section{Detailed Abundances of One Star}

We measured detailed abundances from our HIRES spectrum of star 40 using standard high-resolution abundance analysis techniques. First, we adopted the atomic line list of Kirby \& Cohen (2012). The list is described in detail by Cohen et al. (2003). Second, we fit Voigt profiles to all of the absorption lines in the list that were identifiable. We used local measurements of the spectral continuum within 10 line widths of the line centers. We fit $2 \sigma$ upper limits for undetected lines. We found the upper limit first by calculating $\chi_{\text {flat }}^{2}$ for a flat spectrum. The limit was established as the Voigt profile with $\chi^{2}=\chi_{\text {flat }}^{2}+4$. Table 5 gives the equivalent widths (EWs) of the Voigt profiles along with the abundance determined for each line. For species without line detections, we present the upper limit only on the line that yields the most stringent abundance limit. 
Table 5

Line List with Equivalent Widths

\begin{tabular}{lccccc}
\hline \hline Species & $\begin{array}{c}\text { Wavelength } \\
(\AA)\end{array}$ & $\begin{array}{c}\text { EP } \\
(\mathrm{eV})\end{array}$ & $\log g f$ & $\begin{array}{c}\text { EW } \\
(\mathrm{m} \AA)\end{array}$ & $\epsilon$ \\
\hline $\mathrm{Li}$ I & 6707.760 & 0.000 & -0.002 & $<7.6$ & $<0.65$ \\
$\mathrm{O} \mathrm{I}$ & 6300.304 & 0.000 & -9.780 & $<4.2$ & $<6.77$ \\
$\mathrm{Na} \mathrm{I}$ & 5889.950 & 0.000 & +0.108 & 96.0 & 2.40 \\
$\mathrm{Na} \mathrm{I}$ & 5895.924 & 0.000 & -0.194 & 89.5 & 2.62 \\
$\mathrm{Mg}$ I & 4057.505 & 4.350 & -0.900 & 21.8 & 4.96 \\
$\mathrm{Mg}$ I & 4702.991 & 4.350 & -0.440 & 46.1 & 4.88 \\
$\mathrm{Mg}$ I & 5172.684 & 2.710 & -0.393 & 193.3 & 4.97 \\
$\mathrm{Mg}$ I & 5183.604 & 2.720 & -0.167 & 204.3 & 4.87 \\
$\mathrm{Mg}$ I & 5528.405 & 4.350 & -0.498 & 48.9 & 4.93 \\
$\mathrm{Al} \mathrm{I}$ & 3961.520 & 0.010 & -0.340 & 135.1 & 3.53 \\
$\ldots$ & $\ldots$ & $\cdots$ & $\ldots$ & $\cdots$ & $\cdots$ \\
\hline
\end{tabular}

Note. Wavelengths are in air.

(This table is available in its entirety in machine-readable form.)

Next, we used MOOG to compute abundances with an ATLAS9 model atmosphere (Kurucz 1993; Castelli \& Kurucz 2004). Kirby (2011) constructed a grid of ATLAS9 model atmospheres with a fine spacing in $[\alpha / \mathrm{Fe}]$. We used a model that was interpolated from within that grid. We found $T_{\text {eff }}$, surface gravity, and microturbulence by minimizing the slope of abundance versus excitation potential, minimizing the difference in abundance between $\mathrm{Fe}$ I and Fe II, and minimizing the slope of abundance versus reduced width $(\mathrm{EW} / \lambda)$. This method is known to produce temperatures and surface gravities that are too low (e.g., Thévenin and Idiart 1999). Hence, we used the empirical temperature correction derived by Frebel et al. (2013). This correction raises the temperature computed by the above method. We then remeasured surface gravity and microturbulence by minimizing $\mid \epsilon(\mathrm{Fe}$ I $)-\epsilon(\mathrm{Fe}$ II $) \mid$ and the slope of abundance versus reduced width. Figure 9 shows the excitation potential and reduced width trends. The latter is consistent with zero. The difference in iron abundance from $\mathrm{Fe}$ I and Fe II lines is also zero. The abundance does depend on excitation potential because we applied the Frebel et al. (2013) correction to the temperature. Table 6 gives the abundances from the final iteration of MOOG, referenced to the solar abundances of Asplund et al. (2009).

The stellar parameters measured from HIRES are $T_{\text {eff }}=4816 \quad \mathrm{~K}, \quad \log g=1.64, \quad$ microturbulence $\quad \xi=2.51$ $\mathrm{km} \mathrm{s}^{-1},[\mathrm{Fe} / \mathrm{H}]=-2.92$, and $[\alpha / \mathrm{Fe}]=+0.25$. The temperature we measured from the DEIMOS spectrum is $101 \mathrm{~K}$ higher, and the surface gravity is 0.25 dex higher (see Table 4). The higher temperature led us to measure a metallicity 0.14 dex higher in the DEIMOS spectrum compared to the HIRES spectrum. The individual $[\alpha / \mathrm{Fe}]$ ratios are consistent to within the errors.

The abundances of $\mathrm{Na}, \mathrm{Al}$, and $\mathrm{K}$ have been modified to account for non-local thermodynamic equilibrium (NLTE) corrections. The corrections for the two $\mathrm{Na} \mathrm{D}$ lines are -0.05 dex (Lind et al. 2011). For Al I $\lambda 3962$, we interpolated Andrievsky et al.'s (2008) corrections in $T_{\text {eff }}$ to find +0.51 dex. We estimated the NLTE correction for K I $\lambda 7699$ as -0.21 dex based on stars of very similar stellar parameters from Andrievsky et al. (2010). However, Andrievsky et al. caution that the NLTE correction for potassium should be computed individually for each star's stellar parameters and potassium EW. For example, an extrapolation in metallicity of Ivanova \&
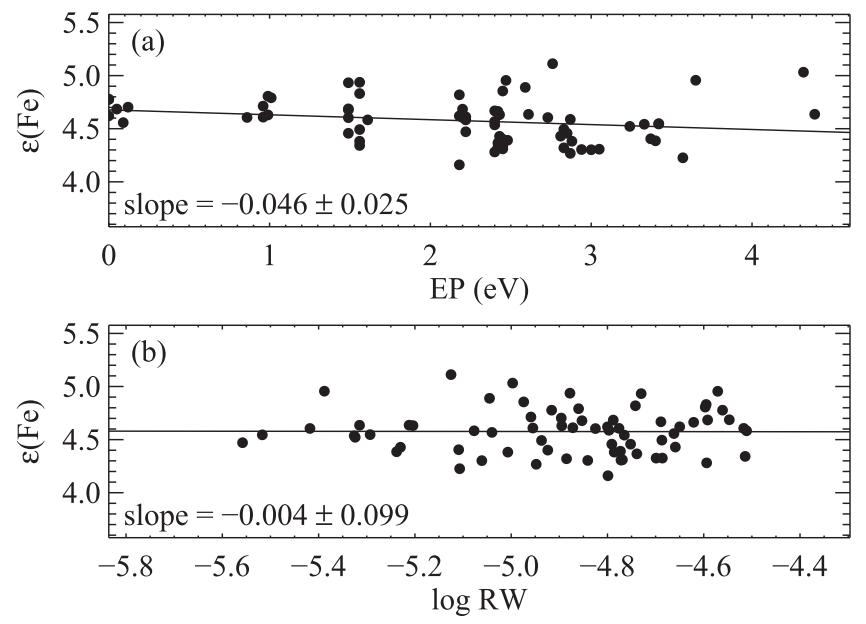

Figure 9. Abundances derived from Fe I lines vs. (a) their excitation potentials and (b) the logarithms of their reduced widths $(\mathrm{EW} / \lambda)$. The solid lines and the figure annotations show the best-fit linear slopes. The excitation balance is not perfect because we applied an empirical correction to the spectroscopic temperature (Frebel et al. 2013).

Table 6

Abundances in Tri II 40

\begin{tabular}{|c|c|c|c|c|c|}
\hline Element & $N$ & $\epsilon$ & {$[\mathrm{X} / \mathrm{H}]$} & {$[\mathrm{X} / \mathrm{Fe}]$} & $\sigma$ \\
\hline $\mathrm{Li}$ I & 1 & $<0.65$ & $<-0.40$ & $<+2.53$ & $\ldots$ \\
\hline $\mathrm{C} \mathrm{I}$ & synth $^{a}$ & 5.42 & -3.01 & -0.09 & 0.15 \\
\hline O I & 1 & $<6.77$ & $<-1.92$ & $<+1.00$ & $\ldots$ \\
\hline $\mathrm{Na} \mathrm{I}{ }^{b}$ & 2 & 2.51 & -3.73 & -0.81 & 0.15 \\
\hline Mg I & 5 & 4.92 & -2.68 & +0.24 & 0.05 \\
\hline $\mathrm{Al} \mathrm{I} \mathrm{I}^{\mathrm{b}}$ & 1 & 3.53 & -2.92 & 0.00 & $\ldots$ \\
\hline Si I & 1 & $<5.35$ & $<-2.16$ & $<+0.76$ & $\ldots$ \\
\hline $\mathrm{K} \mathrm{I}^{\mathrm{b}}$ & 1 & 2.98 & -2.05 & +0.88 & $\ldots$ \\
\hline $\mathrm{Ca} \mathrm{I}$ & 16 & 3.82 & -2.52 & +0.40 & 0.20 \\
\hline Sc II & 5 & 0.53 & -2.62 & +0.30 & 0.09 \\
\hline Ti I & 8 & 2.20 & -2.75 & +0.17 & 0.14 \\
\hline Ti II & 16 & 2.17 & -2.78 & +0.14 & 0.16 \\
\hline V I & 1 & 1.03 & -2.90 & +0.02 & $\ldots$ \\
\hline Cr I & 7 & 2.27 & -3.37 & -0.45 & 0.30 \\
\hline Mn I & 4 & 2.07 & -3.36 & -0.44 & 0.25 \\
\hline $\mathrm{Fe} I$ & 70 & 4.58 & -2.92 & $\ldots$ & 0.21 \\
\hline $\mathrm{Fe}$ II & 13 & 4.58 & -2.92 & $\ldots$ & 0.21 \\
\hline Co I & 1 & 2.19 & -2.79 & +0.13 & $\ldots$ \\
\hline $\mathrm{Ni} \mathrm{I}$ & 4 & 3.82 & -2.40 & +0.53 & 0.30 \\
\hline $\mathrm{Zn} \mathrm{I}$ & 1 & 2.04 & -2.52 & +0.40 & $\ldots$ \\
\hline Sr II & 2 & -1.56 & -4.43 & -1.51 & 0.32 \\
\hline Y II & 1 & $<-1.47$ & $<-3.68$ & $<-0.76$ & $\ldots$ \\
\hline Ba II & 1 & -3.11 & -5.29 & -2.37 & $\ldots$ \\
\hline La II & 1 & $<-1.79$ & $<-2.89$ & $<+0.03$ & $\ldots$ \\
\hline $\mathrm{Ce}$ II & 1 & $<-1.12$ & $<-2.70$ & $<+0.22$ & $\ldots$ \\
\hline Nd II & 1 & $<-1.25$ & $<-2.67$ & $<+0.25$ & $\ldots$ \\
\hline Eu II & 1 & $<-2.38$ & $<-2.90$ & $<+0.02$ & $\ldots$ \\
\hline Dy II & 1 & $<0.17$ & $<-0.93$ & $<+1.99$ & $\ldots$ \\
\hline
\end{tabular}

Notes.

a Measured by spectral synthesis.

b NLTE corrections applied to these species.

Shimanskii's (2000) tabular NLTE corrections suggests that the correction should be -0.49 dex. Weaker lines will be less affected by NLTE corrections. The K I $\lambda 7699$ in star 40 is rather strong for its temperature and metallicity. Thus, the true correction could be stronger than what we have applied. 


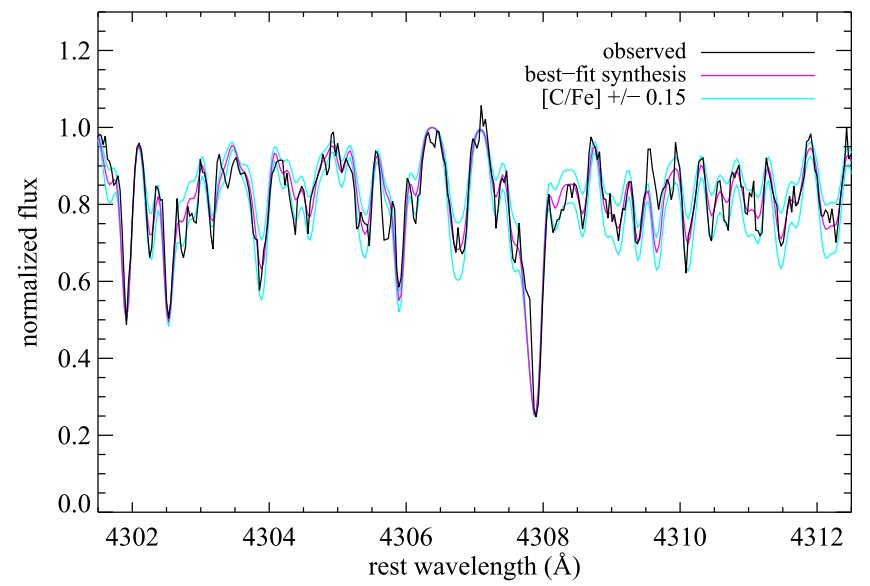

Figure 10. Small region of the Keck/HIRES spectrum, showing the main part of the $\mathrm{G}$ band of the $\mathrm{CH}$ molecule. The magenta line shows the best-fit synthesis, and the cyan lines show the synthesis corresponding to changing the carbon abundance by our quoted error of \pm 0.15 dex.

We measured carbon by synthesis of the Fraunhofer $\mathrm{G}$ band due to the $\mathrm{CH}$ molecule. We adopted the line list of Jorgensen et al. (1996). We fit the spectrum in the region of the $\mathrm{G}$ band by varying the carbon abundance to minimize $\chi^{2}$. Then, following Kirby \& Cohen (2012), we refined the continuum determination by computing the residual between the observed spectrum and the synthesis, fitting a polynomial to it, and then dividing the observed spectrum by that polynomial. We repeated the $\chi^{2}$ minimization and continuum refinement until the carbon abundance did not change between successive iterations. Figure 10 shows the best-fitting spectrum along with spectra with the carbon abundance altered by $\pm 0.15 \mathrm{dex}$, which is the measurement uncertainty that we quote. Table 6 gives the carbon abundance that we measured.

Our high-resolution spectroscopic results for star 40 agree well with those of V17. We determined $T_{\text {eff }}$ to be $16 \mathrm{~K}$ higher, $\log g$ to be 0.16 dex lower, and $\xi$ to be $0.19 \mathrm{~km} \mathrm{~s}^{-1}$ lower than V17. V17 determined $T_{\text {eff }}$ with the infrared flux method, whereas we used excitation balance coupled with Frebel et al's (2013) empirical temperature correction. The close agreement of our results supports the validity of the empirical correction. The metallicity agrees to within 0.05 dex. Most other abundances agree within the error bars with two exceptions. First, V17 measured an upper limit for carbon as $\epsilon(\mathrm{C})<4.7$, whereas we measured $\epsilon(C)=5.42 \pm 0.15$. The difference arises from the spectral features used to measure carbon. We used the G band at $4300 \AA$, but V17 did not have access to that region of the spectrum. Hence, they used weaker $\mathrm{CH}$ features. Figure 10 shows that our detection of carbon is clear. Second, V17 measured $\epsilon(\mathrm{Ti})=2.67 \pm 0.17$, whereas we measured $\epsilon(\mathrm{Ti})=2.18 \pm 0.16$. Our line list for Ti does not overlap with V17 at all. We do not have an explanation for the discrepancy, but we note that our Ti abundances from 24 independent lines have an interquartile range of 0.19 dex and a standard deviation of 0.16 dex. Also, the difference between the average abundance from $\mathrm{Ti}$ I and $\mathrm{Ti}$ II lines is only 0.03 dex.

Star 40 exhibits the hallmark of detailed abundances in a UFD: an extreme deficiency of neutron-capture elements. Our measurement of $[\mathrm{Ba} / \mathrm{Fe}]=-2.4$ is lower than that found in globular clusters (e.g., Ivans et al. 1999; Sneden et al. 2000a, 2000b; Cohen 2011) or in the MW halo (Gratton \& Sneden 1994; Fulbright 2000; Cohen et al. 2004, 2008). Our measurement of $[\mathrm{Sr} / \mathrm{Fe}]=-1.5$ is also very low. Highresolution spectroscopy of most UFD stars shows severe deficiencies in $\mathrm{Ba}$ and other neutron-capture elements (e.g., Frebel et al. 2010a, 2010b, 2014; Koch et al. 2013; Ji et al. 2016b, 2016d). One major exception is the UFD Reticulum II, in which most stars have highly super-solar $[n / \mathrm{Fe}]$ ratios (Ji et al. 2016a, 2016c). One explanation for this neutron-capture enhancement is that Reticulum II experienced a rare event, such as a merger between two neutron stars or a neutron star and a black hole, that produced a great deal of $r$ process material (Freiburghaus et al. 1999; Nishimura et al. 2016). Star 40 in Tri II has not been affected by such an event. Instead, Tri II seems to have lacked a strong source of the $r$ process, similar to most other UFDs.

The abundances of most other elements in star 40 are also typical for dwarf galaxies. The star is enhanced in $\alpha$ elements, consistent with enrichment by one or more core collapse supernovae. Lithium is not detected. Lithium deficiency is expected in giants because they have undergone the first dredge-up, which mixes lithium-depleted material from the interior of the star to the surface (e.g., Pilachowski et al. 1993; Ryan \& Deliyannis 1995; Kirby et al. 2016). The carbon abundance is sub-solar, which is also expected for a red giant above the luminosity function bump in the RGB. Our measurement of $[\mathrm{C} / \mathrm{Fe}]$ in star 40 is consistent with $[\mathrm{C} / \mathrm{Fe}]$ for red giants of similar luminosity in classical dSphs (Kirby et al. 2015b).

V17 pointed out that the potassium abundance seems to be enhanced in star 40. This is especially noteworthy because some globular clusters exhibit an anti-correlation between $\mathrm{Mg}$ and $\mathrm{K}$ abundances. The K-enhanced population in NGC 2419 shows $[\mathrm{K} / \mathrm{Fe}]>+1$ (Cohen et al. 2011; Cohen \& Kirby 2012; Mucciarelli et al. 2012). NGC 2808 contains a sub-population with a much milder $\mathrm{K}$ enrichment $([\mathrm{K} / \mathrm{Fe}] \sim+0.3$, Mucciarelli et al. 2015), slightly larger than the average value for metalpoor halo stars (e.g., Cayrel et al. 2004). Prior to the discovery of $\mathrm{K}$ enhancement in NGC 2419, it was thought that $\mathrm{K}$ was constant within every globular cluster and at most mildly enhanced compared to the Sun (Takeda et al. 2009). We refer the reader to V17 for further discussion of $\mathrm{K}$ in star 40, but we note that the finding of a large potassium abundance depends on the NLTE correction. V17 applied the same correction as we did. Ideally, the correction would be computed by a full NLTE calculation for this star, as pointed out by Andrievsky et al. (2010).

\section{Discussion}

Willman \& Strader (2012) proposed that a galaxy be defined as a stellar system that contains dark matter. One diagnostic of this criterion is the observation of a velocity dispersion or rotation velocity larger than can be explained by the baryonic mass of the galaxy. Alternatively, a galaxy can be identified by circumstantial evidence for dark matter, such as the ability to retain supernova ejecta despite a stellar mass that is too small to prevent the complete escape of such ejecta. Stellar systems that do not pass these tests should instead be described as star clusters. K15a and M16 identified Tri II as a galaxy because they both measured a velocity dispersion significantly in excess of what the stars alone would permit.

We have obtained new medium- and high-resolution spectroscopy of stars in Tri II. We updated our estimate of the stellar velocity dispersion by identifying a binary star 
among the original samples and by revising M16's measurements of individual stellar velocities. The velocities of all but one of the 13 member stars are consistent with a single radial velocity within their $1 \sigma$ error bars, and the other star is consistent at $1.6 \sigma$. In other words, we cannot resolve the velocity dispersion of Tri II. Instead, we give upper limits: $\sigma_{v}<3.4 \mathrm{~km} \mathrm{~s}^{-1}$ (90\% C.L.) and $\sigma_{v}<4.2 \mathrm{~km} \mathrm{~s}^{-1}$ (95\% C.L.). This revision to the velocity dispersion removes the most direct evidence for dark matter in Tri II.

The bulk properties of Tri II's stellar population are not particularly helpful in identifying the system as a star cluster or a galaxy. The half-light radius and absolute luminosity are more consistent with galaxies than star clusters, but the two populations overlap significantly in this space at the low luminosities of a system like Tri II (Drlica-Wagner et al. 2015b; Laevens et al. 2015). Figure 7 shows Tri II's properties derived from the medium-resolution spectroscopy presented in this article. The galaxy lies above the mass-metallicity relation defined by Local Group dwarf galaxies. Both globular clusters and tidally stripped dwarf galaxies occupy this region of the mass-metallicity diagram. The upper limits on the kinematic properties of Tri II are consistent with dwarf galaxies, but they are also consistent with globular clusters, which have low $\mathrm{M} / \mathrm{Ls}$, like stellar populations free of dark matter.

We measured individual metallicities and $[\alpha / \mathrm{Fe}]$ ratios for six stars from medium-resolution spectroscopy. We detected a metallicity dispersion at high significance. However, this conclusion depends on one star, which happens to be the star in a binary system, or another star, which is arguably a nonmember for its large metallicity and large radial displacement from the galaxy center. The detailed abundances of the binary star are unusual. When we fit all available $\mathrm{Mg}, \mathrm{Si}, \mathrm{Ca}$, and $\mathrm{Ti}$ absorption lines in the DEIMOS spectrum simultaneously, we measured $[\alpha / \mathrm{Fe}]=-0.48 \pm 0.15$, which is low for a star with $[\mathrm{Fe} / \mathrm{H}]=-1.91 \pm 0.11$. However, our measurement of the $\mathrm{Mg}$ abundance by itself is somewhat larger: $[\mathrm{Mg} / \mathrm{Fe}]=+0.21 \pm 0.28$. Frustratingly, V17 measured significantly different abundances of this star from a highresolution but low-S $/ \mathrm{N}$ spectrum: $[\mathrm{Fe} / \mathrm{H}]=-2.5 \pm 0.2$ and $[\mathrm{Mg} / \mathrm{Fe}]=-0.7 \pm 0.3$. The conflicting results do not lend confidence to the abundance measurements of this star. However, the detection of a metallicity dispersion is secure either if star 46 has $[\mathrm{Fe} / \mathrm{H}]=-1.9$ or if star 31 is a member.

Our high-resolution spectrum of the brightest known member star in Tri II shows exceptionally low abundances of $\mathrm{Sr}$ and $\mathrm{Ba}$. Deficiencies in neutron-capture elements are the hallmark signature of detailed abundances in UFDs. Star clusters show $[\mathrm{Sr} / \mathrm{Fe}]$ and $[\mathrm{Ba} / \mathrm{Fe}]$ ratios close to solar, and MW halo stars with $[n / \mathrm{Fe}] \lesssim-2$ are extremely rare. The abundances in star 40 in Tri II are $[\mathrm{Sr} / \mathrm{Fe}]=-1.5$ and $[\mathrm{Ba} / \mathrm{Fe}]=-2.4$. Thus, the deficiency in neutron-capture elements in this single star may very well be the strongest evidence in favor of classifying Tri II as a UFD rather than a star cluster.

We thank D. Stern, S. Hemmati, and D. Masters for observing the Trillc slitmask.

We are grateful to the many people who have worked to make the Keck Telescope and its instruments a reality and to operate and maintain the Keck Observatory. The authors wish to extend special thanks to those of Hawaiian ancestry on whose sacred mountain we are privileged to be guests. Without their generous hospitality, none of the observations presented herein would have been possible.

Facility: Keck:II (DEIMOS).

\section{References}

Andrievsky, S. M., Spite, M., Korotin, S. A., et al. 2008, A\&A, 481, 481 Andrievsky, S. M., Spite, M., Korotin, S. A., et al. 2010, A\&A, 509, A88 Asplund, M., Grevesse, N., Sauval, A. J., \& Scott, P. 2009, ARA\&A, 47, 481 Castelli, F., \& Kurucz, R. L. 2004, arXiv:astro-ph/0405087

Cayrel, R., Depagne, E., Spite, M., et al. 2004, A\&A, 416, 1117

Chambers, K. C., Magnier, E. A., Metcalfe, N., et al. 2016, arXiv:1612.05560 Cohen, J. G. 2011, ApJL, 740, L38

Cohen, J. G., Christlieb, N., McWilliam, A., et al. 2004, ApJ, 612, 1107

Cohen, J. G., Christlieb, N., McWilliam, A., et al. 2008, ApJ, 672, 320

Cohen, J. G., Christlieb, N., Qian, Y.-Z., \& Wasserburg, G. J. 2003, ApJ, 588,1082

Cohen, J. G., Huang, W., \& Kirby, E. N. 2011, ApJ, 740, 60

Cohen, J. G., \& Kirby, E. N. 2012, ApJ, 760, 86

Cooper, M. C., Newman, J. A., Davis, M., Finkbeiner, D. P., \& Gerke, B. F. 2012, Spec2d: DEEP2 DEIMOS Spectral Pipeline, Astrophysics Source Code Library, ascl:1203.003

Dekel, A., \& Woo, J. 2003, MNRAS, 344, 1131

Demarque, P., Woo, J.-H., Kim, Y.-C., \& Yi, S. K. 2004, ApJS, 155, 667 Drlica-Wagner, A., Albert, A., Bechtol, K., et al. 2015a, ApJL, 809, L4

Drlica-Wagner, A., Bechtol, K., Rykoff, E. S., et al. 2015b, ApJ, 813, 109

Duchêne, G., \& Kraus, A. 2013, ARA\&A, 51, 269

Faber, S. M., \& Lin, D. N. C. 1983, ApJL, 266, L17

Faber, S. M., Phillips, A. C., Kibrick, R. I., et al. 2003, Proc. SPIE, 4841,1657

Flewelling, H. A., Magnier, E. A., Chambers, K. C., et al. 2016, arXiv:1612. 05243

Frebel, A., Casey, A. R., Jacobson, H. R., \& Yu, Q. 2013, ApJ, 769, 57

Frebel, A., Kirby, E. N., \& Simon, J. D. 2010a, Natur, 464, 72

Frebel, A., Simon, J. D., Geha, M., \& Willman, B. 2010b, ApJ, 708, 560

Frebel, A., Simon, J. D., \& Kirby, E. N. 2014, ApJ, 786, 74

Freiburghaus, C., Rosswog, S., \& Thielemann, F.-K. 1999, ApJL, 525, L121

Fulbright, J. P. 2000, AJ, 120, 1841

Geha, M., Brown, T. M., Tumlinson, J., et al. 2013, ApJ, 771, 29

Geha, M., Willman, B., Simon, J. D., et al. 2009, ApJ, 692, 1464

Genina, A., \& Fairbairn, M. 2016, MNRAS, 463, 3630

Girardi, L., Grebel, E. K., Odenkirchen, M., \& Chiosi, C. 2004, A\&A, 422, 205

Gratton, R., Sneden, C., \& Carretta, E. 2004, ARA\&A, 42, 385

Gratton, R. G., \& Sneden, C. 1994, A\&A, 287, 927

Gustafsson, B., Edvardsson, B., Eriksson, K., et al. 2008, A\&A, 486, 951

Ivanova, D. V., \& Shimanskii, V. V. 2000, ARep, 44, 376

Ivans, I. I., Sneden, C., Kraft, R. P., et al. 1999, AJ, 118, 1273

Ji, A. P., Frebel, A., Chiti, A., \& Simon, J. D. 2016a, Natur, 531, 610

Ji, A. P., Frebel, A., Ezzeddine, R., \& Casey, A. R. 2016b, ApJL, 832, L3

Ji, A. P., Frebel, A., Simon, J. D., \& Chiti, A. 2016c, ApJ, 830, 93

Ji, A. P., Frebel, A., Simon, J. D., \& Geha, M. 2016d, ApJ, 817, 41

Jorgensen, U. G., Larsson, M., Iwamae, A., \& Yu, B. 1996, A\&A, 315, 204

Kaiser, N., Burgett, W., Chambers, K., et al. 2010, Proc. SPIE, 7733, 0

Kirby, E. N. 2011, PASP, 123, 531

Kirby, E. N., Boylan-Kolchin, M., Cohen, J. G., et al. 2013a, ApJ, 770, 16

Kirby, E. N., \& Cohen, J. G. 2012, AJ, 144, 168

Kirby, E. N., Cohen, J. G., Guhathakurta, P., et al. 2013b, ApJ, 779, 102

Kirby, E. N., Cohen, J. G., Simon, J. D., \& Guhathakurta, P. 2015a, ApJL, 814, L7

Kirby, E. N., Guhathakurta, P., Bolte, M., Sneden, C., \& Geha, M. C. 2009, ApJ, 705, 328

Kirby, E. N., Guhathakurta, P., Simon, J. D., et al. 2010, ApJS, 191, 352

Kirby, E. N., Guhathakurta, P., \& Sneden, C. 2008, ApJ, 682, 1217

Kirby, E. N., Guhathakurta, P., Zhang, A. J., et al. 2016, ApJ, 819, 135

Kirby, E. N., Guo, M., Zhang, A. J., et al. 2015b, ApJ, 801, 125

Kirby, E. N., Simon, J. D., \& Cohen, J. G. 2015c, ApJ, 810, 56

Koch, A., Feltzing, S., Adén, D., \& Matteucci, F. 2013, A\&A, 554, A5

Koch, A., Hansen, T., Feltzing, S., \& Wilkinson, M. I. 2014, ApJ, 780, 91

Koposov, S. E., Casey, A. R., Belokurov, V., et al. 2015, ApJ, 811, 62

Koposov, S. E., Gilmore, G., Walker, M. G., et al. 2011, ApJ, 736, 146

Kurucz, R. 1993, ATLAS9 Stellar Atmosphere Programs and 2 km/s Grid. Kurucz CD-ROM No. 13 (Cambridge, MA: Smithsonian Astrophysical Observatory), 13

Laevens, B. P. M., Martin, N. F., Ibata, R. A., et al. 2015, ApJL, 802, L18

Larson, R. B. 1974, MNRAS, 169, 229 
Lind, K., Asplund, M., Barklem, P. S., \& Belyaev, A. K. 2011, A\&A, 528, A103

Martin, N. F., Ibata, R. A., Collins, M. L. M., et al. 2016, ApJ, 818, 40

McConnachie, A. W. 2012, AJ, 144, 4

Minor, Q. E. 2013, ApJ, 779, 116

Mucciarelli, A., Bellazzini, M., Ibata, R., et al. 2012, MNRAS, 426, 2889

Mucciarelli, A., Bellazzini, M., Merle, T., et al. 2015, ApJ, 801, 68

Newman, J. A., Cooper, M. C., Davis, M., et al. 2013, ApJS, 208, 5

Nishimura, N., Wanajo, S., Sekiguchi, Y., et al. 2016, JPhCS, 665, 012059

Nomoto, K., Tominaga, N., Umeda, H., Kobayashi, C., \& Maeda, K. 2006, NuPhA, 777, 424

Pilachowski, C. A., Sneden, C., \& Booth, J. 1993, ApJ, 407, 699

Robin, A. C., Reylé, C., Derrière, S., \& Picaud, S. 2003, A\&A, 409, 523

Ryan, S. G., \& Deliyannis, C. P. 1995, ApJ, 453, 819

Schlegel, D. J., Finkbeiner, D. P., \& Davis, M. 1998, ApJ, 500, 525

Simon, J. D., Drlica-Wagner, A., Li, T. S., et al. 2015, ApJ, 808, 95

Simon, J. D., \& Geha, M. 2007, ApJ, 670, 313

Simon, J. D., Geha, M., Minor, Q. E., et al. 2011, ApJ, 733, 46
Simon, J. D., Li, T. S., Drlica-Wagner, A., et al. 2016, AAS Journals, submitted (arXiv:1610.05301)

Skillman, E. D., Kennicutt, R. C., \& Hodge, P. W. 1989, ApJ, 347, 875

Sneden, C., Johnson, J., Kraft, R. P., et al. 2000a, ApJL, 536, L85

Sneden, C., Pilachowski, C. A., \& Kraft, R. P. 2000b, AJ, 120, 1351

Sohn, S. T., Majewski, S. R., Muñoz, R. R., et al. 2007, ApJ, 663, 960

Starkenburg, E., Hill, V., Tolstoy, E., et al. 2010, A\&A, 513, A34

Takeda, Y., Kaneko, H., Matsumoto, N., et al. 2009, PASJ, 61, 563

Thévenin, F., \& Idiart, T. P. 1999, ApJ, 521, 753

Tonry, J. L., Stubbs, C. W., Lykke, K. R., et al. 2012, ApJ, 750, 99

Vargas, L. C., Geha, M., Kirby, E. N., \& Simon, J. D. 2013, ApJ, 767, 134

Venn, K. A., Starkenburg, E., Malo, L., Martin, N., \& Laevens, B. P. M. 2017, MNRAS, 466, 3741

Walker, M. G., Mateo, M., Olszewski, E. W., et al. 2006, AJ, 131, 2114

Walker, M. G., Mateo, M., Olszewski, E. W., et al. 2007, ApJL, 667, L53

Willman, B., \& Strader, J. 2012, AJ, 144, 76

Wolf, J., Martinez, G. D., Bullock, J. S., et al. 2010, MNRAS, 406, 1220

Woosley, S. E., \& Weaver, T. A. 1995, ApJS, 101, 181 